ARTICLE

\title{
Virus-specific memory $T$ cell responses unmasked by immune checkpoint blockade cause hepatitis
}

James A. Hutchinson (1) ${ }^{1 凶}$, Katharina Kronenberg${ }^{1}$, Paloma Riquelme ${ }^{1}$, Jürgen J. Wenzel (10 ${ }^{2}$, Gunther Glehr (10 ${ }^{3}$, Hannah-Lou Schilling (10 ${ }^{1}$, Florian Zeman ${ }^{4}$, Katja Evert ${ }^{5}$, Martin Schmiedel ${ }^{6}$, Marion Mickler ${ }^{7}$, Konstantin Drexler ${ }^{7}$, Florian Bitterer ${ }^{1}$, Laura Cordero', Lukas Beyer ${ }^{8}$, Christian Bach ${ }^{9}$, Josef Koestler ${ }^{2}$, Ralph Burkhardt ${ }^{10}$, Hans J. Schlitt (10 1, Dirk Hellwig (1) 6, Jens M. Werner ${ }^{1}$, Rainer Spang ${ }^{3}$, Barbara Schmidt (10 2, Edward K. Geissler ${ }^{1,11}$ \& Sebastian Haferkamp ${ }^{7}$

Treatment of advanced melanoma with combined PD-1/CTLA-4 blockade commonly causes serious immune-mediated complications. Here, we identify a subset of patients predisposed to immune checkpoint blockade-related hepatitis who are distinguished by chronic expansion of effector memory $C D 4^{+} T$ cells ( $T_{E M}$ cells). Pre-therapy $C D 4^{+} T_{E M}$ cell expansion occurs primarily during autumn or winter in patients with metastatic disease and high cytomegalovirus (CMV)-specific serum antibody titres. These clinical features implicate metastasisdependent, compartmentalised $C M V$ reactivation as the cause of $C D 4^{+} T_{E M}$ expansion. Pre-therapy $C D 4^{+} \mathrm{T}_{E M}$ expansion predicts hepatitis in $C M V$-seropositive patients, opening possibilities for avoidance or prevention. 3 of 4 patients with pre-treatment $C D 4^{+} \mathrm{T}_{\mathrm{EM}}$ expansion who received $\alpha$ PD- 1 monotherapy instead of $\alpha$ PD- $1 / \alpha C T L A-4$ therapy remained hepatitis-free. 4 of 4 patients with baseline $C D 4^{+} T_{E M}$ expansion given prophylactic valganciclovir and $\alpha \mathrm{PD}-1 / \alpha \mathrm{CTLA}-4$ therapy remained hepatitis-free. Our findings exemplify how pathogen exposure can shape clinical reactions after cancer therapy and how this insight leads to therapeutic innovations.

\footnotetext{
${ }^{1}$ Department of Surgery, University Hospital Regensburg, Regensburg, Germany. ${ }^{2}$ Institute of Clinical Microbiology and Hygiene, University Hospital Regensburg, Regensburg, Germany. ${ }^{3}$ Institute of Functional Genomics and Statistical Bioinformatics, University of Regensburg, Regensburg, Germany. ${ }^{4}$ Center for Clinical Studies, University Hospital Regensburg, Regensburg, Germany. ${ }^{5}$ Institute of Pathology, University Hospital Regensburg, Regensburg, Germany. ${ }^{6}$ Department of Nuclear Medicine, University Hospital Regensburg, Regensburg, Germany. ${ }^{7}$ Department of Dermatology, University Hospital Regensburg, Regensburg, Germany. ${ }^{8}$ Institute of Radiology, University Hospital Regensburg, Regensburg, Germany. ${ }^{9}$ Department of Medicine $V$, University Hospital Erlangen, Erlangen, Germany. ${ }^{10}$ Institute of Clinical Chemistry and Laboratory Medicine, University Hospital Regensburg, Regensburg, Germany. ${ }^{11}$ Personalised Tumour Therapy, Fraunhofer Institute for Experimental Medicine and Toxicology, Regensburg, Germany.

凶email: james.hutchinson@ukr.de
} 
T reatment with anti-programmed cell death protein-1 ( $\alpha \mathrm{PD}$ 1) alone or combined with anti-cytotoxic T-lymphocyteassociated protein 4 (aCTLA-4) has greatly improved the prognosis of metastatic melanoma with a substantial fraction of patients achieving long-lasting partial or complete clinical responses $^{1-6}$. The principal limitation of $\alpha \mathrm{PD}-1 /$ CCTLA- 4 dual therapy is the relatively high rate of immune-related complications, which commonly include colitis, hepatitis and thyroiditis, but may also include pneumonitis, myocarditis and encephalitis ${ }^{7,8}$. Such adverse events range from mild reactions to life-threatening presentations, but are nevertheless clinically significant incidents that influence patient management ${ }^{9,10}$. Avoidance of complications has led to a somewhat complicated treatment decisionmaking process (Fig. 1a) that seeks to balance individual risk and capacity to tolerate adverse reactions against the superior clinical responses achieved with dual $\alpha$ PD-1/aCTLA-4 therapy over aPD-1 monotherapy ${ }^{11-13}$.

Our aetiological understanding of colitis, hepatitis and thyroiditis caused by immune checkpoint blockade remains incomplete $^{14}$; however, these treatment-related conditions are clearly different from known autoimmune diseases ${ }^{15,16}$. Such adverse reactions are usually attributed to generalised dysregulation of $\mathrm{T}$ cells ${ }^{17,18}$ or autoantibodies ${ }^{15}$. Here, we explore a different view-namely, that individual patients are predisposed to particular treatment-related complications by virtue of pathogenspecific memory $\mathrm{T}$ cells present in their repertoire before immune checkpoint blockade is given.

Cytomegalovirus (CMV) seroprevalance amongst European adults is over $50 \%$. Primary CMV infection usually presents during young adulthood as an acute febrile illness, after which life-long latency is established. The exact sites and mechanisms of CMV latency are not fully resolved, but myelomonocytic cells in blood and tissues are major reservoirs ${ }^{19}$. CMV reactivation characteristically follows immune compromise caused by immunosuppressive therapy or other viral infection ${ }^{20}$. Clinical manifestations of CMV reactivation are variable, ranging from classic febrile illness to tissue-invasive disease presenting as colitis, hepatitis, pneumonitis or encephalitis ${ }^{21}$. Reactivation of CMV causes cellular injury through the cytopathic effect of viral replication, but more conspicuously through triggering $\mathrm{T}$ cell immunity. Pneumonitis in transplant recipients illustrates how CMV-reactive $\mathrm{T}$ cells provoke extensive, life-threatening inflammation despite low-level viral replication ${ }^{22}$. Even in anatomical locations from which $\mathrm{T}$ cells are normally excluded, such as the retina, $\mathrm{T}$ cell-mediated responses significantly contribute to CMV-related disease. Thus, low-level and compartmentalised reactivation of $\mathrm{CMV}$ can be responsible for serious acute immunopathology in humans ${ }^{23-25}$.

Cycles of CMV latency and reactivation lead to a now wellrecognised phenomenon of memory inflation, which is typified by failure of primed $\mathrm{CMV}$-specific $\mathrm{CD}^{+} \mathrm{T}$ cell populations to contract and give rise to central memory $\mathrm{T}$ cells ${ }^{26}$. Instead, a dominant pool of effector memory $\mathrm{CD}^{+}{ }^{+} \mathrm{T}$ cells that does not become functionally exhausted is maintained ${ }^{27}$. Such inflationary $\mathrm{CD}^{+} \mathrm{T}$ cells achieve high frequencies over time, sustained through repetitive stimulation by viral reactivation or long-term persistence of viral antigens ${ }^{28}$. Memory inflation is described as an exclusive feature of $\mathrm{CD} 8^{+}$effector $\mathrm{T}$ cell responses, although durable expansion of $\mathrm{CMV}$-specific $\mathrm{CD} 4^{+} \mathrm{T}$ cells can occur in transplant recipients ${ }^{29}$.

This study identifies pre-therapy expansion of $\mathrm{CD} 4^{+}$effector memory $\mathrm{T}$ cells $\left(\mathrm{T}_{\mathrm{EM}}\right)$ in $\mathrm{CMV}$-infected patients with metastatic melanoma as a prognostic marker of aPD-1/aCTLA-4-related hepatitis. Opting for aPD-1 monotherapy or prophylactic valganciclovir in CMV $\mathrm{IgG}^{+}$patients with unresectable metastatic disease and pre-treatment expansion of $\mathrm{CD} 4^{+} \mathrm{T}_{\mathrm{EM}}$ cells reduces their risk of hepatitis. We conclude that sub-clinical reactivation of CMV plays a previously unsuspected role in the immunopathogenesis of aPD-1/aCTLA-4-related hepatitis in a subgroup of patients.

\section{Results}

Clinical features do not predict aPD-1/aCTLA-4-related hepatitis. If immune-related complications of aPD-1/aCTLA-4 therapy resulted solely from treatment-induced immunological effects $^{30,31}$, we might expect hepatitis, colitis and thyroiditis to coincide often ${ }^{32}$ (Fig. 1b). However, no such associations were found in a cohort of 89 patients with metastatic melanoma treated with aPD-1/aCTLA-4 dual therapy (Fig. 1c, Supplementary Fig. 1 and Supplementary Tables 1-3). This raises the possibility that some individuals are predisposed to particular adverse reactions.

In this study, we focused on factors that predispose to $\alpha \mathrm{PD}-1 /$ aCTLA-4-related hepatitis. Except for patients who developed hepatitis being generally younger (Supplementary Table 4) no associations were found between incidence of hepatitis and pretreatment clinical variables (Supplementary Tables 4 and 5). Conventional biochemical markers of liver dysfunction, systemic inflammation or tumour burden did not discriminate between patients who did or did not develop hepatitis (Fig. 1d-j). We found no associations between incidence of hepatitis and seropositivity for hepatitis $\mathrm{B}$ virus (HBV), hepatitis $\mathrm{C}$ virus (HCV) or hepatitis $\mathrm{E}$ virus (HEV; Fig. 1k-m). Incidence of hepatitis was not greater with more rounds of aPD-1/aCTLA-4 therapy; in fact, as immune-related complications are reason to withhold treatment, patients who developed hepatitis tended to receive fewer rounds of $a \mathrm{PD}-1 /$ aCTLA-4 (Fig. 1n). Autoimmune hepatitis-related autoantibodies were not associated with risk of hepatitis (Supplementary Table 6). In short, case history and routine clinical investigations did not predict hepatitis after dual therapy in our study cohort.

$\mathrm{CD4}^{+} \mathrm{T}_{\mathrm{EM}}$ cell expansion predicts aPD-1/aCTLA-4-related hepatitis. We next profiled circulating leucocyte subsets immediately prior to start of dual therapy in a training set of $n=44$ patients with unresectable metastatic melanoma (Supplementary Figs. 2-8). Comparing the frequency of 50 principal leucocyte subsets between patients who subsequently did $(n=18)$ or did not $(n=26)$ develop hepatitis revealed significant overrepresentation of $\mathrm{CD}^{+} \mathrm{T}_{\mathrm{EM}}$ cells associated with hepatitis (Fig. 2a, b). This observation was confirmed in a validation set $(n=45)$ of similar patients (Fig. 2c). Baseline expansion of $\mathrm{CD}^{+}$ $\mathrm{T}_{\mathrm{EM}}$ cells was a fair discriminator $(\mathrm{AUROC}=0.706)$ of patients who did or did not later develop hepatitis in the validation set (Fig. 2d). Strikingly, CD4 ${ }^{+} \mathrm{T}_{\mathrm{EM}}$ cell frequencies in all patients with completely resected disease were normally distributed with no extreme cases of baseline $\mathrm{CD} 4^{+} \mathrm{T}_{\mathrm{EM}}$ cell expansion, allowing us to set a threshold below which $99 \%$ of patients should fall (Fig. 2e). Applying this stringent cut-off to our validation cohort of patients with unresectable metastatic disease (Fig. 2f) allowed us to correctly predict hepatitis in 6 of 6 patients with $\mathrm{CD} 4^{+}$ $\mathrm{T}_{\mathrm{EM}} \geq 21 \%$ (specificity $=100 \%$ ) and correctly identify 6 of 20 patients who developed hepatitis (sensitivity $=30.0 \%$ ). $\mathrm{CD} 4^{+}$ $\mathrm{T}_{\mathrm{EM}}{ }^{221 \%}$ patients were not predisposed to colitis (Fig. $2 \mathrm{~g}$ ), thyroiditis or superior clinical responses to therapy (Supplementary Table 7). Hepatitis was not more severe in $\mathrm{CD}^{+} \mathrm{T}_{\mathrm{EM}} \geq 21 \%$ patients compared to $\mathrm{CD} 4^{+} \mathrm{T}_{\mathrm{EM}}<21 \%$ patients (Fig. $2 \mathrm{~h}$ ) and no difference in time-to-first presentation was observed (Fig. 2i). Hence, baseline $\mathrm{CD} 4^{+} \mathrm{T}_{\mathrm{EM}}$ expansion is a specific, but relatively insensitive prognostic marker of individuals who are predisposed to hepatitis after aPD-1/aCTLA-4 therapy. Owing to its low sensitivity, classifying patients as $\mathrm{CD}^{+}{ }^{+} \mathrm{T}_{\mathrm{EM}}<21 \%$ has limited clinical utility; however, specifically identifying cases at high-risk 
a

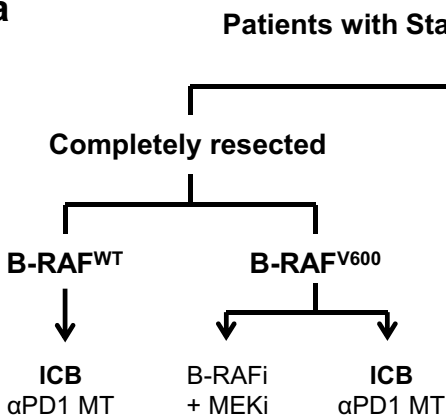

b

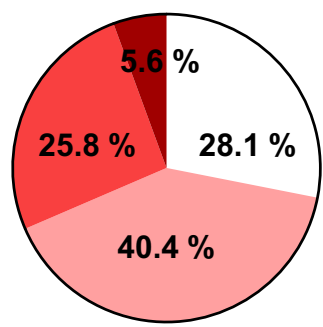

$\square$ No complications

$\square 1$ complication

2 complications

3 complications
ICB = Immune Checkpoint Blockade MT = Monotherapy, DT = Dual Therapy
Unresectable metastatic disease

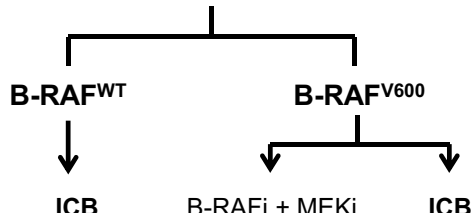

MT: aPD1 or DT: $\alpha$ PD1 + aCTLA4

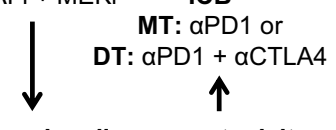

Progressive disease or toxicity c

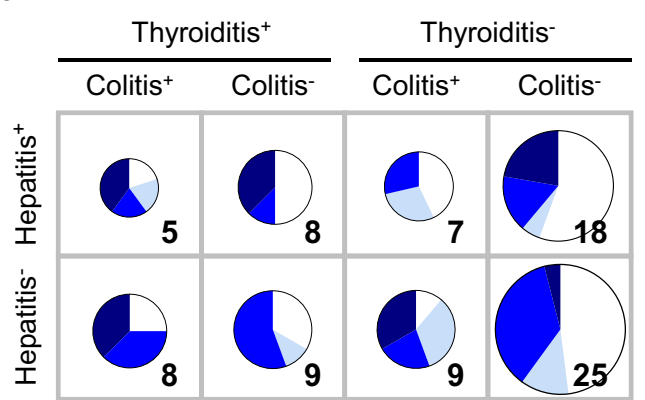

d

d $p=1.000$

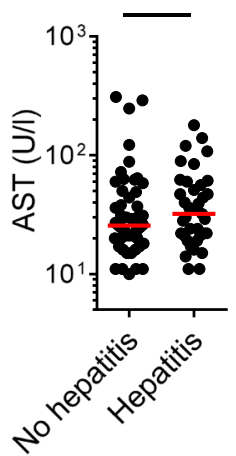

h

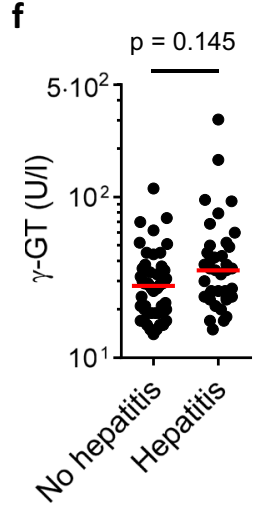

k

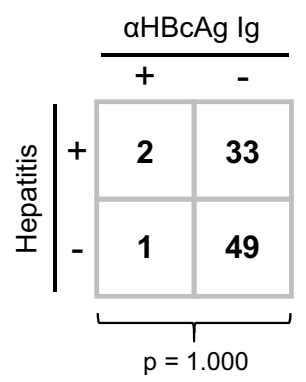

g $\quad p=1.000$
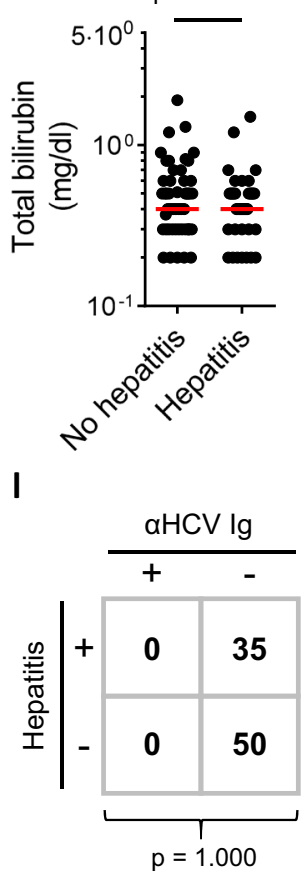

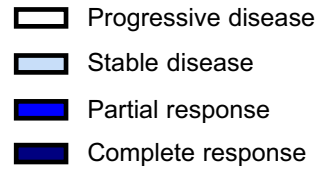

$p=0.073$

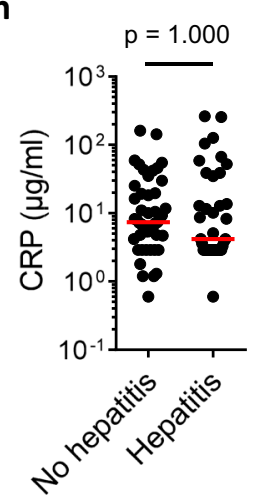

m
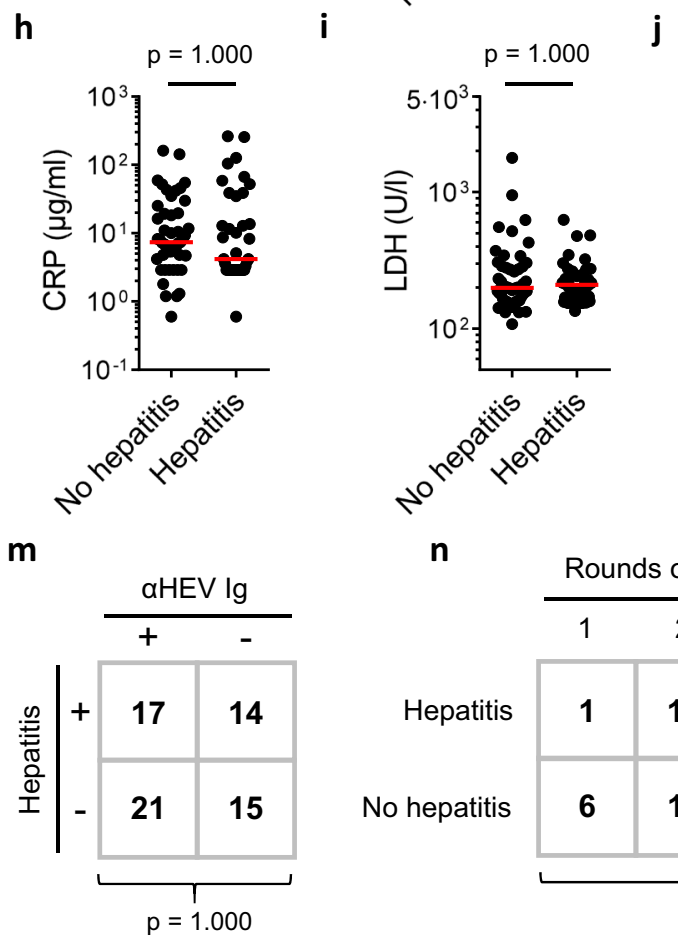

n e

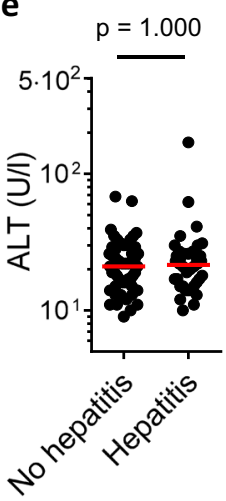

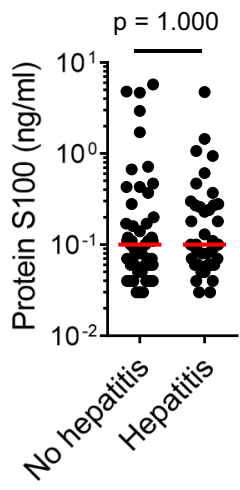

Rounds of DT administered

\begin{tabular}{c|c|c|c|c|}
\cline { 2 - 4 } & \multicolumn{1}{c}{1} & 2 & \multicolumn{1}{c}{3} & \multicolumn{1}{c}{4} \\
\cline { 2 - 5 } Hepatitis & 1 & 12 & 10 & 15 \\
\cline { 2 - 5 } No hepatitis & 6 & 10 & 6 & 29 \\
\cline { 2 - 5 } & \multicolumn{4}{|c|}{$p=0.059$}
\end{tabular}

of hepatitis before treatment is useful from a research perspective because we can then prospectively study the immunological basis of their predisposition.

Opting for aPD-1 monotherapy reduces risk of hepatitis. Although aPD-1 monotherapy achieves fewer sustained clinical responses in patients with metastatic melanoma, it causes fewer adverse reactions than $\alpha \mathrm{PD}-1 / \alpha \mathrm{CTLA}-4$ dual therapy ${ }^{1}$. Therefore, there is a clear rationale for avoiding aPD-1/aCTLA-4 dual therapy in patients at risk of hepatitis. Four $\mathrm{CD}^{+} \mathrm{T}_{\mathrm{EM}} \geq 21 \%$ patients with metastasic disease presented during our study, but were electively treated with aPD-1 monotherapy (Fig. 2j). 
Fig. 1 Individual predisposition to hepatitis after $\boldsymbol{\alpha P D}-\mathbf{1 / \alpha C T L A - 4}$ treatment. a Individualised treatment of melanoma is guided by tumour staging, presence of B-RAF mutations and fitness-for-toxicity. $\mathbf{b}$ Colitis, hepatitis and thyroiditis are common immune-related complications of dual therapy with Nivolumab plus Ipilimumab; $31.4 \%$ of patients experienced two or more of these immune-related adverse reactions $(n=89)$. c Colitis, hepatitis and thyroiditis occurred independently and were not significantly associated with clinical response $(n=89 ;$ F.E). d-h Patients who developed hepatitis of any grade following dual therapy lacked biochemical signs of liver inflammation before treatment. In particular, no clinically meaningful differences in plasma levels of $\mathbf{d}$ aspartate transaminase (AST; $n=87 ;$ M.W.; Bonferroni-corrected $p$-value, $m=5$ ), e alanine transaminase (ALT; $n=89 ;$ M.W.; Bonferronicorrected $p$-value, $m=5)$, f gamma glutamyl transaminase ( $\gamma$-GT; $n=89 ;$ M.W.; Bonferroni-corrected $p$-value, $m=5), \mathbf{g}$ total bilirubin $(n=87$; M.W.; Bonferroni-corrected $p$-value, $m=5$ ), or $\mathbf{h}$ C-reactive protein (CRP; $n=85$; M.W.; Bonferroni-corrected $p$-value, $m=5$ ) were observed between patients who developed hepatitis and those who did not. Median values are indicated by a red line. $\mathbf{i}$, $\mathbf{j}$ Biochemical markers of tumour burden were not different between patients who developed hepatitis and those who did not. i Pre-treatment levels of lactate dehydrogenase $(n=89 ;$ M.W.; Bonferroni-corrected $p$-value, $m=4)$. Median values are indicated by a red line. $\mathbf{j}$ Pre-treatment levels of protein $\mathrm{S} 100(n=89 ; \mathrm{M} . \mathrm{W}$.; Bonferroni-corrected $p$-value, $m=4)$. $\mathbf{k}-\mathbf{m}$ No association was observed between seropositivity for $\mathbf{k}$ hepatitis $B$ virus core antigen ( $\mathrm{HBcAg} ; n=85 ;$ F.E.; Bonferroni-corrected $p$-value, $m=3$ ), I hepatitis $\mathrm{C}$ virus (HCV; $n=85$; F.E.; Bonferroni-corrected $p$-value, $m=3$ ), or $\mathbf{m}$ hepatitis E virus (HEV; $n=67$; F.E.; Bonferroni-corrected $p$-value, $m=3$ ) and development of hepatitis following dual therapy. $\mathbf{n}$ No association was observed between rounds of $\alpha$ PD- $1 / \alpha C T L A-4$ administered and development of hepatitis ( $n=89$; M.W.).

Whereas 12 of $12 \mathrm{CD}^{+} \mathrm{T}_{\mathrm{EM}} \geq 21 \%$ patients treated with aPD-1/ aCTLA-4 dual therapy developed hepatitis, 3 of 4 CD4 $+\mathrm{T}_{\mathrm{EM}} \geq 21 \%$ patients who received $\alpha \mathrm{PD}-1$ monotherapy remained hepatitis free. Reducing the incidence hepatitis by opting for aPD-1 monotherapy hints that CTLA-4 blockade may be mechanistically important for development of hepatitis in $\mathrm{CD}^{+}$ $\mathrm{T}_{\mathrm{EM}} \geq 21 \%$ patients ${ }^{33}$.

$\mathrm{CD4}^{+} \mathrm{T}_{\mathrm{EM}}$ frequency is an independent predictor of hepatitis. We next investigated possible causes of baseline $\mathrm{CD}^{+}{ }^{+} \mathrm{T}_{\mathrm{EM}}$ expansion. To establish CD4 ${ }^{+} \mathrm{T}_{\mathrm{EM}}$ expansion as an independent risk factor, we first sought to exclude possible confounding covariables. Baseline CD4 ${ }^{+} \mathrm{T}_{\mathrm{EM}} \geq 21 \%$ was not related to sex, age or body-mass index (Supplementary Table 7 and Fig. 3a, b). No significant correlation was observed between baseline $\mathrm{CD} 4^{+}$ $\mathrm{T}_{\mathrm{EM}} \%$ and serum levels of C-reactive protein (CRP), aspartate aminotransferase (AST), alanine aminotransferase (ALT), gamma-glutamyl transferase ( $\gamma$-GT) or total bilirubin (Fig. $3 c-g$ ). Because glucose metabolism provides a reliable measure of liver inflammation $^{34}$, we were able to further rule out liver inflammation prior to therapy as a predisposing cause of $\mathrm{CD}^{+}$ $\mathrm{T}_{\mathrm{EM}} \geq 21 \%$ hepatitis by estimating hepatic glucose uptake in patients who underwent ${ }^{18} \mathrm{~F}$-fluoro-2-deoxy-D-glucose ( $\left.{ }^{18} \mathrm{~F}-\mathrm{FDG}\right)$ $\mathrm{PET} / \mathrm{CT}$ studies as part of routine tumour staging shortly before starting immunotherapy (Fig. $3 \mathrm{~h}$ ). This retrospective comparison showed ${ }^{18} \mathrm{~F}-\mathrm{FDG}$ standard uptake ratios $\left(\mathrm{SUR}_{\text {mean }}\right.$ liver $)$ were higher in $\mathrm{CD} 44^{+} \mathrm{T}_{\mathrm{EM}}<21 \%$ patients than $\mathrm{CD} 4^{+} \mathrm{T}_{\mathrm{EM}} \geq 21 \%$ patients with hepatitis (Fig. 3i). Therefore, our biochemical and radiological investigations argue against pre-treatment liver inflammation as a common cause for baseline $\mathrm{CD} 4^{+} \mathrm{T}_{\mathrm{EM}}$ expansion and treatment-related hepatitis.

Because $\mathrm{CD}^{+} \mathrm{T}_{\mathrm{EM}}$ expansion is principally a feature of metastatic melanoma (Fig. 2e), we next asked whether $\mathrm{CD}^{+}$ $\mathrm{T}_{\mathrm{EM}} \%$ was somehow associated with tumour burden. No relationship was found between $\mathrm{CD}^{+} \mathrm{T}_{\mathrm{EM}} \%$ and the number of organs containing metastases, presence of hepatic metastases or serum lactate dehydrogenase (LDH) and protein-S100 serum levels (Fig. 3j-m and Supplementary Table 7). There was a tendency for $\mathrm{CD}^{+} \mathrm{T}_{\mathrm{EM}} \geq 21 \%$ patients to have received previous therapy of any type (including BRAFi/MEKi or Talimogen laherparepvec) but besides implying a longer disease course before starting dual therapy, we see no mechanistic interpretation because these drugs share no common pharmacological action (Fig. 3n). In short, we found no obvious clinical association to explain the predisposition of $\mathrm{CD}^{+} \mathrm{T}_{\mathrm{EM}} \geq 21 \%$ patients to hepatitis.
$\mathrm{CD4}^{+} \mathrm{T}_{\mathrm{EM}}$ cell expansion is associated with chronic $\mathrm{T}$ cell activation. Although $\mathrm{CD}^{+}{ }^{+} \mathrm{T}$ cell counts were not different between $\mathrm{CD}^{+} \mathrm{T}_{\mathrm{EM}}<21 \%$ and $\mathrm{CD} 4{ }^{+} \mathrm{T}_{\mathrm{EM}^{2}} \geq 21 \%$ patients (Supplementary Table 7), there were significantly fewer circulating naïve $\mathrm{T}$ cells in $\mathrm{CD}^{+} \mathrm{T}_{\mathrm{EM}} \geq 21 \%$ patients (Fig. 4a). This reduction was complemented by increased absolute numbers of $\mathrm{CD} 4^{+} \mathrm{T}_{\mathrm{EM}}$ and $\mathrm{T}_{\mathrm{EMRA}}$ cells (Fig. 4b, c) whereas central memory $\mathrm{T}$ cell $\left(\mathrm{T}_{\mathrm{CM}}\right)$ counts tended to be lower in $\mathrm{CD}^{+} \mathrm{T}_{\mathrm{EM}} \geq 21 \%$ patients (Fig. $4 \mathrm{~d}$ ). We next defined other leucocyte subsets that correlated with $\mathrm{CD}^{+}{ }^{+} \mathrm{T}_{\mathrm{EM}} \%$ in both our training and validation cohorts (Fig. 4e). Most covariant populations were $\mathrm{T}$ cells, particularly $\mathrm{CD} 4^{+} \mathrm{T}$ cell subsets. Changes in the CD4 ${ }^{+} \mathrm{T}$ cell compartment of $\mathrm{T}_{\mathrm{EM}} \geq 21 \%$ patients were consistent with chronic activation, particularly downregulation of CD27 and upregulation of CD57, HLA-DR and CD279 expression (Fig. 4f-i). Similar differences were detected in $\mathrm{CD}^{+} \mathrm{T}$ cells (Supplementary Fig. 9). Notably, CD4 ${ }^{+} \mathrm{T}_{\mathrm{EM}} \geq 21 \%$ patients tended to have higher frequencies of $\mathrm{CD} 160^{+} \mathrm{CD} 244^{+}$ exhausted $\mathrm{CD}^{+} \mathrm{T}$ cells than $\mathrm{CD} 4^{+} \mathrm{T}_{\mathrm{EM}}{ }^{<21 \%}$ patients (Fig. 4j).

Most adults are persistently infected with Torque Teno Virus (TTV), a non-enveloped, circular, single-stranded DNA virus of the Anellovirus family that causes no apparent disease. $\mathrm{T}$ cell immunity controls TTV replication in healthy people, whereas TTV copy number in immunocompromised individuals is increased; therefore, TTV load in plasma is a useful marker of immune competence. Despite accumulating chronically activated and exhausted T cells, TTV load in $\mathrm{CD} 4^{+} \mathrm{T}_{\mathrm{EM}} \geq 21 \%$ versus $\mathrm{CD} 4^{+}$ $\mathrm{T}_{\mathrm{EM}}{ }^{<21 \%}$ patients was not different, implying that $\mathrm{CD} 4{ }^{+} \mathrm{T}_{\mathrm{EM}} \geq 21 \%$ patients are not generally immunocompromised (Fig. 4k).

$\mathrm{CD}^{+} \mathrm{T}_{\mathrm{EM}}$ cell expansion correlates with immunity to $\mathrm{CMV}$. To briefly summarise, baseline expansion of $\mathrm{CD} 4^{+} \mathrm{T}_{\mathrm{EM}}$ cells identifies a subset of patients with metastasic melanoma who are predisposed to $\mathrm{aPD}-1 / \mathrm{aCTLA}-4$-related hepatitis. These patients show signs of persistent or recurrent $\mathrm{CD} 4^{+}$and $\mathrm{CD} 8^{+}$ $\mathrm{T}$ cell responses ${ }^{35}$, but are otherwise clinically and immunologically unremarkable. It is usually very difficult to isolate factors responsible for such a non-specific immunological picture ${ }^{36}$. Fortunately, a surprising pattern emerged from our dataset: $\mathrm{CD}^{+} \mathrm{T}_{\mathrm{EM}} \geq 21 \%$ patients mostly presented between September and February (Fig. 5a). This seasonal influence was apparent over all 4 years of the study, leading us to think that CD4 ${ }^{+} \mathrm{T}_{\mathrm{EM}}$ expansion might be driven by a pathogen. Active infections with hepatitis viruses, including $\mathrm{HBV}$ and $\mathrm{HCV}$, are relative contraindications to $\alpha \mathrm{PD}-1 / \mathrm{\alpha CTLA}-4$ therapy (Supplementary Table 7), so we were forced to consider possible involvement of other hepatotropic viruses, such as HEV and herpesgroup viruses. 
a

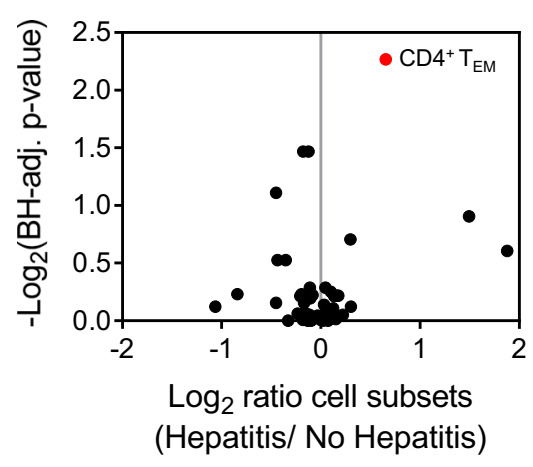

b

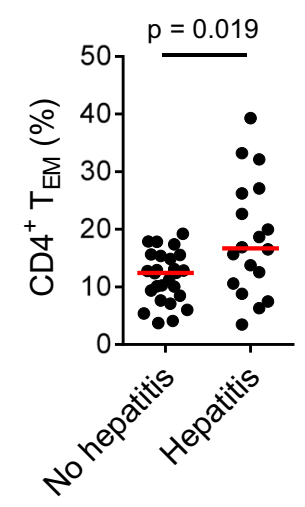

C

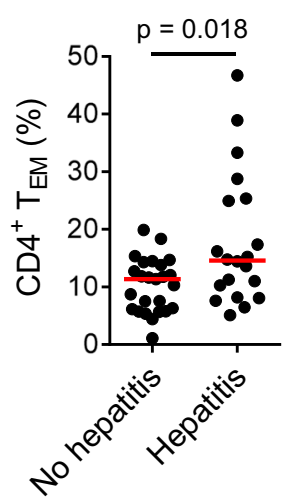

d

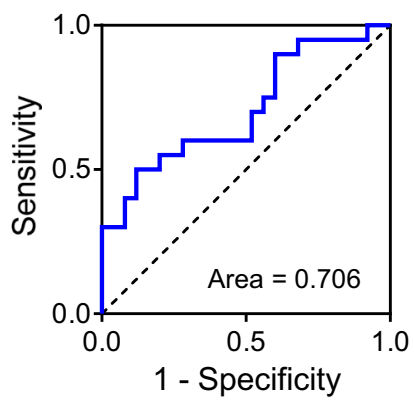

e

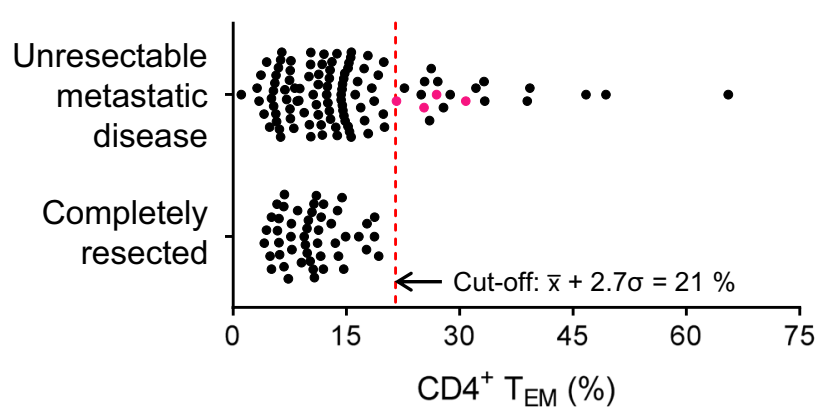

f

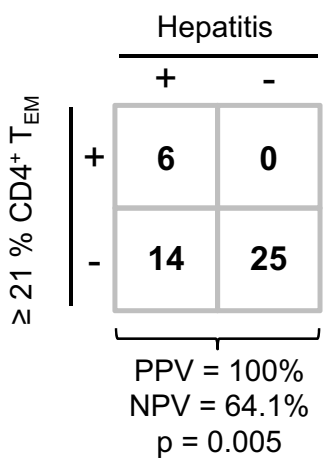

g

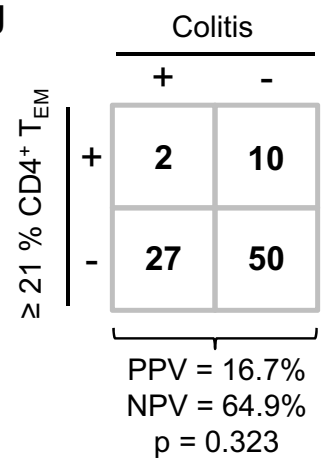

h

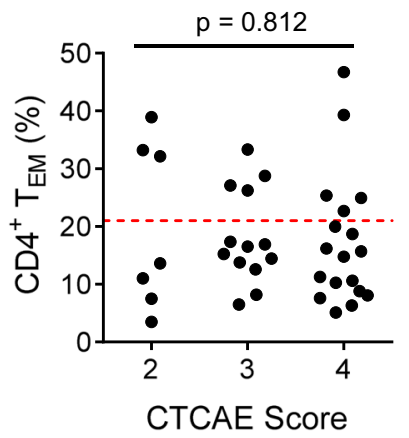

i

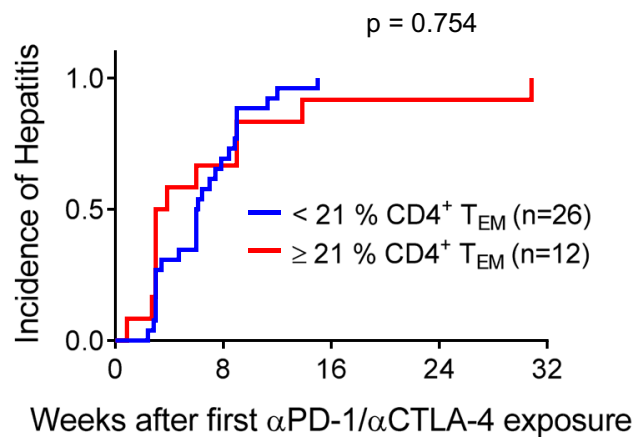

j

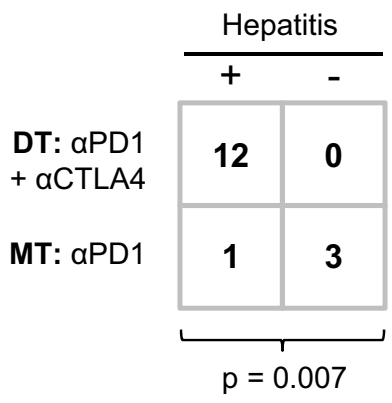

Fig. 2 Circulating CD4 ${ }^{+} \mathbf{T}$ effector memory cell frequency predicts hepatitis after Nivolumab plus Ipilimumab treatment. a Peripheral blood samples were collected from melanoma patients with metastatic disease receiving $\alpha$ PD- $1 / \alpha C T L A-4$ therapy immediately before administration of the first dose $(n=89)$. Leucocyte subsets differentially represented in patients with or without hepatitis were identified in a randomly assigned training set (B.H.corrected $t$-tests; $n=44 ; m=50 ; F D R=0.25$ ). Red dots indicate significantly differently represented subsets. Example gating strategies for analysis of flow cytometry data are provided as Supplementary Figs. 2-8. b CD4 ${ }^{+} \mathrm{T}_{\mathrm{EM}} \%$ in training set patients with or without treatment-related hepatitis $(n=44$; M.W.). Median values are indicated by a red line. c CD4 ${ }^{+} T_{E M} \%$ in validation set patients with or without treatment-related hepatitis $(n=45 ;$ M.W.). d ROC analysis of CD4 ${ }^{+} \mathrm{T}_{\mathrm{EM}} \%$ as a discriminatory marker for treatment-related hepatitis in the validation set $(n=45)$. e Comparison of the bimodal distribution of $\mathrm{CD}_{4}+\mathrm{T}_{\mathrm{EM}} \%$ in patients with unresectable metastatic disease $(n=107)$ and the normal distribution $(n=49 ; \mathrm{K} 2=2.79 ; p=0.248)$ of $C D 4+T_{E M} \%$ in patients with completely resected tumours. A cut-off of CD4 $+T_{E M} \geq 21 \%$ was set (indicated by a dashed red line) below which $99 \%$ of completely resected tumour cases should fall. Four pink points represent $C D 4+\mathrm{T}_{\mathrm{EM}} \geq 21 \%$ patients with metastatic disease who were electively treated with $\alpha$ PD-1 monotherapy. $\mathbf{f}$ In the validation set, $68.9 \%$ patients were correctly classified using a cut-off of CD4 ${ }^{+} \mathrm{T}_{\mathrm{EM}} \geq 21 \%$, whereas $55.6 \%$ were correctly classified under the no-information model $\left(n=45\right.$; F.E.). $\mathbf{g}$ CD4 ${ }^{+} \mathrm{T}_{\mathrm{EM}} \geq 21 \%$ is not a marker of predisposition to $\alpha$ PD- $1 / \alpha C T L A-4-r e l a t e d$ colitis $(n=89$; F.E.). h CD4 ${ }^{+} \mathrm{T}_{\mathrm{EM}} \geq 21 \%$ patients did not experience more severe hepatitis than $\mathrm{CD}^{+} \mathrm{T}_{\mathrm{EM}}{ }^{<2 \%}$ patients $(n=38$; F.E.). Dashed red line indicates a cut-off of CD4 ${ }^{+} \mathrm{T}_{\mathrm{EM}} \geq 21 \%$. i Time-to-first presentation of hepatitis was not different between CD4 ${ }^{+} \mathrm{T}_{\mathrm{EM}} \geq 21 \%(n=12)$ and $\mathrm{T}_{\mathrm{EM}}<21 \%(n=26)$ patients (log-rank). j Twelve of 12 patients with unresectable metastatic melanoma and CD4+ $\mathrm{T}_{\mathrm{EM}} \geq 21 \%$ developed hepatitis after $\alpha \mathrm{PD}-1 / \alpha \mathrm{CTLA}-4$ dual therapy. By contrast, 3 of $4 \mathrm{CD}^{+}{ }^{+} \mathrm{T}_{\mathrm{EM}} \geq 21 \%$ patients treated with $\alpha \mathrm{PD}-1$ monotherapy did not develop hepatitis (F.E.; $p=0.007$ ).

Latent CMV infection is prevalent amongst European adults and involves many organs, including liver. Compartmentalised reactivation of CMV infection occurs in immunocompromised individuals and those with concomitant viral upper respiratory tract infections ${ }^{20}$. In our patients, we found a strong association between serum CMV-specific IgG antibody levels and $\mathrm{CD} 4^{+} \mathrm{T}_{\mathrm{EM}}$ status (Fig. 5b). Furthermore, T cell reactivity against CMV pp65 peptides in an IFN- $\gamma$ enzyme-linked immunospot (ELISPOT) assay was tightly associated with $\mathrm{CD} 4^{+} \mathrm{T}_{\mathrm{EM}}$ status (Fig. 5c). These results led us to hypothesise a causal relationship between compartmentalised, sub-clinical CMV reactivation in liver and chronic enrichment of $\mathrm{CD} 4^{+} \mathrm{T}_{\mathrm{EM}}$ cells in blood. 
a

- Hepatitis: $\mathrm{CD}^{+} \mathrm{T}_{\mathrm{EM}} \geq 21 \%$

- Hepatitis: $\mathrm{CD}^{+} \mathrm{T}_{\mathrm{EM}}<21 \%$

- No hepatitis

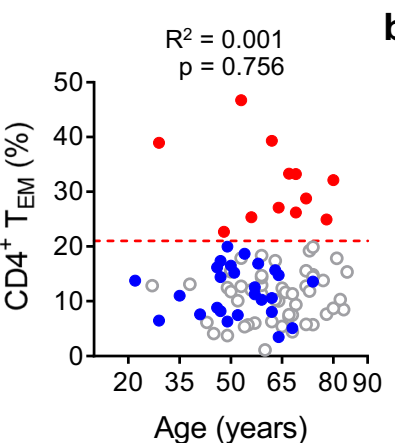

d

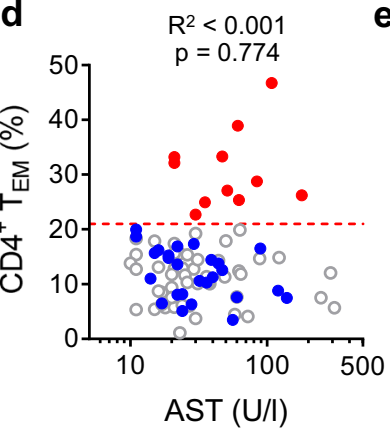

h

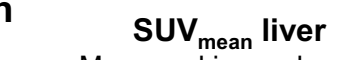

Measured in a spherical region of interest in right lobe
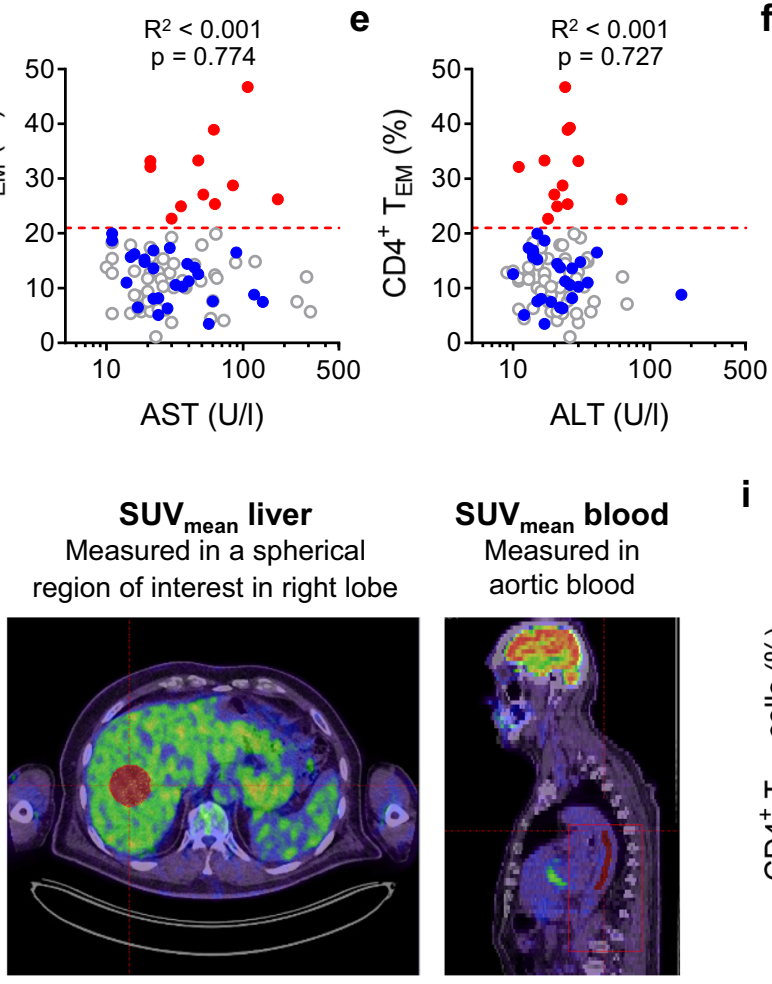

$\mathrm{ALT}(\mathrm{U} / \mathrm{I})$

SUV $_{\text {mean }}$ blood

Measured in aortic blood

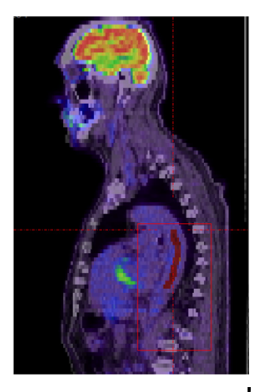

SUR $_{\text {mean }}$ liver $=\left(\mathrm{T}_{0} / \mathrm{T}\right) \cdot\left(\mathrm{SUV}_{\text {mean }}\right.$ liver $/ \mathrm{SUV}_{\text {mean }}$ blood $)$

i
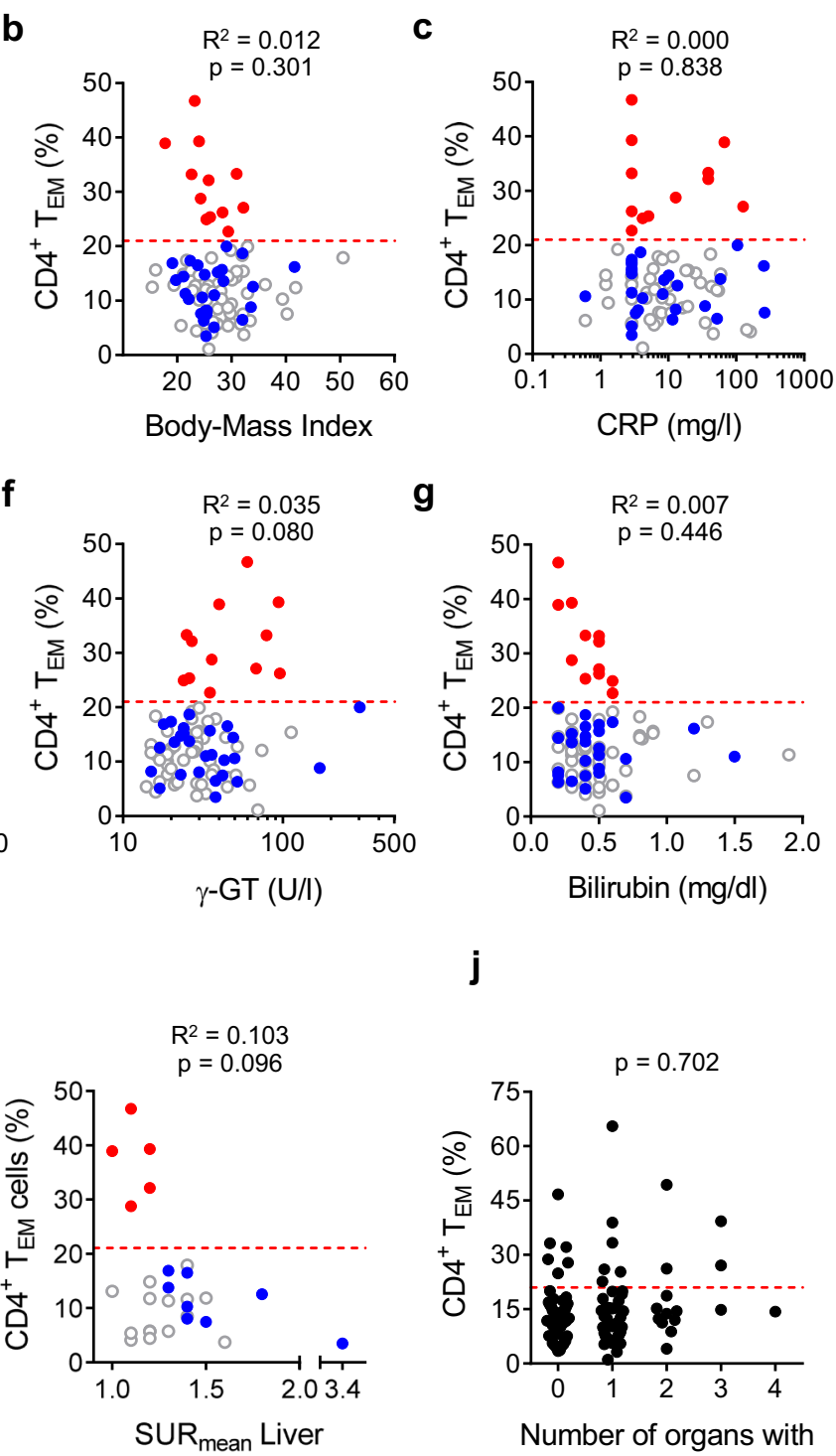

Number of organs with one or more metastases $\geq 1.5 \mathrm{~cm}$ diameter

n

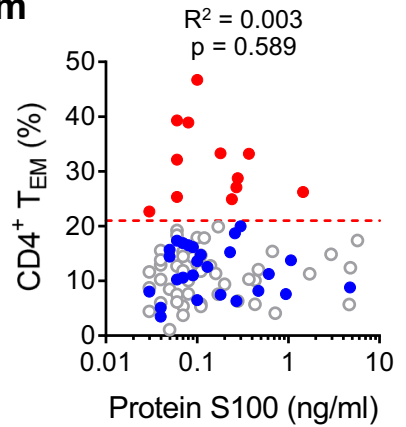

Because baseline $\mathrm{CD} 4^{+} \mathrm{T}_{\mathrm{EM}}$ cell expansion was mainly a feature of $\mathrm{CMV} \mathrm{IgG}^{+}$patients (Fig. 5d), we next asked whether incorporating CMV IgG status could improve our predictive model. When considering only $\mathrm{CMV} \mathrm{IgG}^{+}$cases, $\mathrm{CD} 4^{+} \mathrm{T}_{\mathrm{EM}} \%$ was a more discriminatory marker of hepatitis (Fig. 5e).
Therefore, using our training set filtered for $\mathrm{CMV} \mathrm{IgG}^{+}$cases, we revised our cut-off to $\mathrm{CD} 4^{+} \mathrm{T}_{\mathrm{EM}} \geq 16 \%$ in $\mathrm{CMV} \mathrm{IgG}^{+}$cases in order to achieve a better compromise between specificity and sensitivity (Supplementary Fig. 10). The new cut-off performed well in the validation set with a correct classification rate of $81.0 \%$ 
Fig. 3 CD4 ${ }^{+} \mathbf{T}_{\mathrm{EM}}$ frequency is an independent predictor of $\boldsymbol{\alpha P D}-\mathbf{1} / \mathbf{\alpha C T L A - 4}-$ related hepatitis. a CD4 $+\mathrm{T}_{\mathrm{EM}} \%$ did not correlate with age $(n=89 ;$ Pearson). Dashed red line indicates a cut-off of $\mathrm{CD} 4^{+} \mathrm{T}_{\mathrm{EM}} \geq 21 \% . \mathbf{b} \mathrm{CD} 4^{+} \mathrm{T}_{\mathrm{EM}} \%$ did not correlate with body mass index $(n=89 ; \mathrm{Pearson})$. c $\mathrm{CD} 4{ }^{+} \mathrm{T}_{\mathrm{EM}} \%$ did not correlate with C-reactive protein (CRP) levels $\left(n=85\right.$; Pearson). d CD4 $+\mathrm{T}_{E M} \%$ did not correlate with aspartate aminotransferase (AST) levels ( $n=87$; Pearson). e CD4 $+\mathrm{T}_{\mathrm{EM}} \%$ did not correlate with alanine aminotransferase (AST) levels $\left(n=89 ;\right.$ Pearson). f CD4+ $\mathrm{T}_{\mathrm{EM}} \%$ did not correlate with gamma-glutamyltransferase $\left(\boldsymbol{\gamma}\right.$-GT) levels $\left(n=89\right.$; Pearson). $\mathbf{g}$ CD4 ${ }^{+} \mathrm{T}_{\mathrm{EM}} \%$ did not correlate with total bilirubin levels $(n=89 ;$ Pearson). $\mathbf{h}$ Glucose metabolism measured by ${ }^{18} \mathrm{~F}$-fluoro-2-deoxy-D-glucose ( $\left.{ }^{18} \mathrm{~F}-\mathrm{FDG}\right)$ uptake in PET/CT studies is a quantitative marker of liver inflammation. Calculating standard uptake ratio (SUR mean Liver) allows accurate quantification of ${ }^{18} \mathrm{~F}-\mathrm{FDG}$ uptake in liver by correcting for differential clearance of tracer from blood and liver parenchyma. Higher SUR mean Liver values indicate more severe inflammation. $T_{0}=$ reference time point at 75 min post-injection and $T=$ actual scan time. i SUR mean Liver was lower in CD4 $+T_{E M} \geq 21 \%$ patients than $C D 4+T_{E M}<21 \%$ patients who developed hepatitis indicating less inflammation at baseline in the $C D 4^{+} T_{E M} \geq 21 \%$ subgroup $\left(n=28\right.$; Pearson). $\mathbf{j} C D 4^{+} T_{E M} \geq 21 \%$ was not associated with the number of organs containing one or more metastases $\geq 1.5 \mathrm{~cm}$ in diameter $\left(n=103\right.$; F.E.). $\mathbf{k}$ CD4 ${ }^{+} \mathrm{T}_{\mathrm{EM}} \geq 21 \%$ was not associated with the presence of hepatic metastases $\left(n=103 ; \mathrm{F} . \mathrm{E}\right.$.). I CD4 ${ }^{+}$ $\mathrm{T}_{\mathrm{EM}} \%$ did not correlate with lactate dehydrogenase (LDH) levels $\left(n=88\right.$; Pearson). $\mathbf{m} \mathrm{CD} 4{ }^{+} \mathrm{T}_{\mathrm{EM}} \%$ did not correlate with protein S100 levels ( $n=88$; Pearson). $\mathbf{n} C D 4+\mathrm{T}_{\mathrm{EM}} \geq 21 \%$ was not associated with any prior therapy $(n=89 ; \mathrm{F} . \mathrm{E}$.$) .$

(positive predictive value $(\mathrm{PPV})=85.7 \%$ and negative predictive value $(\mathrm{NPV})=78.6 \%)$ compared to $42.9 \%$ under the noinformation model.

For descriptive purposes, we can consider the distribution of patients from the combined training and validation sets according to CMV IgG and $\mathrm{CD}^{+}{ }^{+} \mathrm{T}_{\mathrm{EM}}$ status (Fig. 5f). Our predictive model explains the occurrence of hepatitis in 34 of $89(38.2 \%)$ of all patients (red boxes). Two of 17 (12\%) CMV $\mathrm{IgG}^{+} \mathrm{CD}^{+}$ $\mathrm{T}_{\mathrm{EM}} \geq 16 \%$ patients (green box) did not develop hepatitis: one of these received four rounds of aPD-1/aCTLA-4 therapy and registered no complications; however, the other received only 1 round of $\alpha \mathrm{PD}-1 / \mathrm{aCTLA}-4$ before treatment with prednisolone from weeks 2 to 7 for colitis. We speculate 4 of $23 \mathrm{CMV} \mathrm{IgG}^{+}$ $\mathrm{CD}^{+} \mathrm{T}_{\mathrm{EM}}<16 \%$ patients who were incorrectly predicted as hepatitis-negative (pink box) were sampled at an early stage of $\mathrm{CD} 4{ }^{+} \mathrm{T}_{\mathrm{EM}}$ cell expansion and our test was not sensitive enough to discriminate the change. Our model does not explain why 19 of $49 \mathrm{CMV} \mathrm{IgG}^{-}$patients developed hepatitis (blue box) but we believe these represent an aetiologically distinct subset.

CMV-reactive $\mathrm{CD4}^{+} \mathrm{T}$ cells are enriched in $\mathrm{CD4}^{+} \mathrm{T}_{\mathrm{EM}}$ high patients. We next asked whether $\mathrm{CMV}$-reactive $\mathrm{CD} 4^{+} \mathrm{T}$ cells were enriched in patients with baseline $\mathrm{CD} 4^{+} \mathrm{T}_{\mathrm{EM}}$ expansion. Peripheral blood mononuclear cells (PBMC) were stimulated in culture for $18 \mathrm{~h}$ with CMV lysates before IFN- $\gamma$, TNFa, IL-17, IL4 and CD69 expression was measured by flow cytometry (Fig. 6a and Supplementary Figs. 11 and 12). To detect hyporesponsive CMV-specific T cells, paired cultures were also stimulated in the presence of neutralising antibodies against PD-1 and CTLA-4. In contrast to $\mathrm{CMV} \mathrm{IgG}^{-}$and $\mathrm{CMV} \mathrm{IgG}{ }^{+} \mathrm{CD}^{+} \mathrm{T}_{\mathrm{EM}}<16 \%$ patients, we readily detected IFN- $\gamma$-producing, CMV-reactive $\mathrm{CD} 4^{+}$ $\mathrm{T}$ cells in CMV IgG ${ }^{+} \mathrm{CD}^{+} \mathrm{T}_{\mathrm{EM}^{2}} \geq 16 \%$ patients (Fig. 6b). This difference implies a higher number of circulating IFN- $\gamma$-producing, CMV-reactive CD $4^{+} \mathrm{T}$ cells (Fig. 6c). Proportions of CMVreactive $\mathrm{CD}^{+}{ }^{+} \mathrm{T}$ cells expressing IL-17, IL-4 or only TNFa were too low to quantify accurately.

Valganciclovir reverses aPD-1/aCTLA-4-related hepatitis. Strong associations between $\mathrm{T}$ cell immunity against CMV, expansion of $\mathrm{CD}^{+} \mathrm{T}_{\mathrm{EM}}$ and risk of treatment-related hepatitis imply an underlying pathogenic mechanism; however, to demonstrate a causal relationship, we needed to establish that inhibiting CMV replication was effective in treating or preventing hepatitis. Valganciclovir is an anti-viral agent licensed for therapy and prophylaxis of CMV infections in immunocompromised patients. It is a widely used and safe drug with a well-known and broadly acceptable profile of adverse effects. Therefore, we next gave valganciclovir treatment to two patients with aPD-1/ aCTLA-4-related hepatitis.
The first patient was a comparatively fit 54-year-old man with a new diagnosis of metastatic melanoma (Fig. 7a). Staging CT revealed multiple lung and bone metastases, but no metastasis to liver or lymph nodes. At baseline, the patient was $\mathrm{CMV}-\mathrm{IgG}^{+}$and $\mathrm{CD}^{+} \mathrm{T}_{\mathrm{EM}}=18.7 \%$ (Fig. $7 \mathrm{~b}$ ). Nivolumab plus Ipilimumab were given at 0,3 and 6 weeks. A fourth round of $\alpha$ PD-1/aCTLA-4 at week 9 was omitted owing to joint pain and the patient received two rounds of Denosumab ( $\alpha$ RANKL). Nivolumab monotherapy was administered in weeks 12, 16, 20 and 24. Staging CT at week 14 showed a partial clinical response with significant regression of pulmonary metastases; hence, checkpoint blockade was of clear clinical benefit.

The patient received a dose of $\alpha \mathrm{PD}-1$ monotherapy in week 32 . Four weeks later, he presented in clinic with grade 4 hepatitis. Strong reactions against pp65 and IE-1 peptides were measured by ELISPOT. Accordingly, the patient was treated with $900 \mathrm{mg} /$ day valganciclovir plus prednisolone. Liver function tests (LFTs) returned to normal, so over the subsequent 2 weeks, prednisolone was weaned and then valganciclovir was withdrawn. Seven days later, the patient returned to clinic with deranged LFTs. Valganciclovir and prednisolone were both reinstated, leading to a rapid normalisation of LFTs. Two days later, a for-cause liver biopsy was performed (Fig. 7c-e). The tissue was essentially normal, although a few dying hepatocytes and isolated lymphocytes were observed in the portal areas. Immunohistochemical staining for CMV and nested CMV PCR from fixed biopsy material were negative. The patient remained clinically stable for 9 days, so he was again weaned from prednisolone by the start of week 43; however, on this occasion, $900 \mathrm{mg}$ /day valganciclovir treatment was continued. After complete withdrawal of prednisolone, while still receiving valganciclovir, the patient's LFTs remained stable for a further 4 weeks. At the start of week 47, valganciclovir was withdrawn for a second time. Subsequently, the patient did not develop hepatitis and required no further steroids. The patient received further doses of aPD-1 monotherapy in weeks 54 and 58 without signs of recurrent liver injury. To us, this suggests the $\mathrm{T}$ cell antigens driving hepatitis in this patient were absent after valganciclovir treatment. In our clinical opinion, despite the absence of detectable virus, the patient's fast recovery and unusual relapse of hepatitis are best explained by introduction, withdrawal and reintroduction of valganciclovir.

The second patient was a 49-year-old man who had an uncomplicated first year after aPD-1/aCTLA- 4 and had been receiving Encorafenib/Binimetinib therapy for 7 weeks when he developed hepatitis in week 67 (Fig. 8a, b). Hepatitis failed to resolve despite withdrawal of B-RAFi/MEKi and escalating doses of prednisolone. $900 \mathrm{mg} /$ day valganciclovir was introduced at the start of week 72 and a for-cause biopsy was performed on the same day (Fig. 8b). Four days later, the patient started a course of $1 \mathrm{mg} /$ day mycophenolate mofetil. Strikingly, AST, ALT and $\gamma$-GT 
a

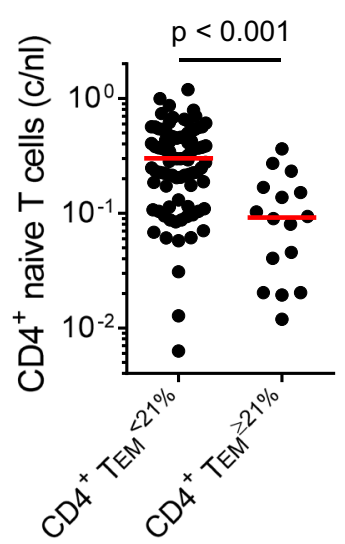

e

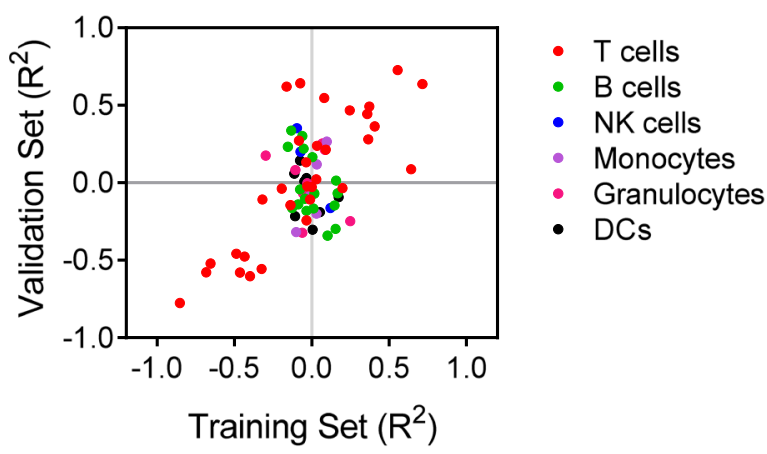

h

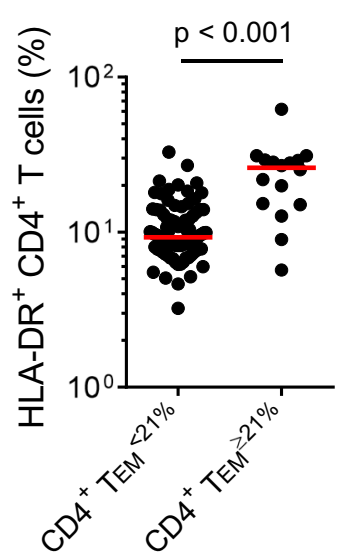

b

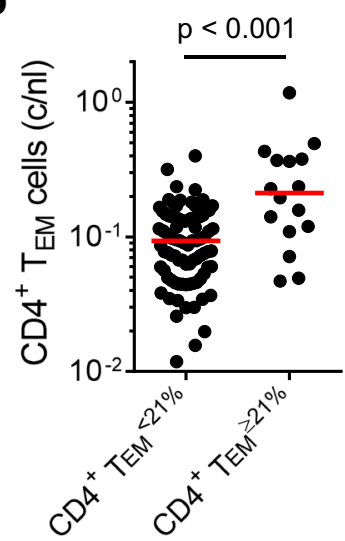

i

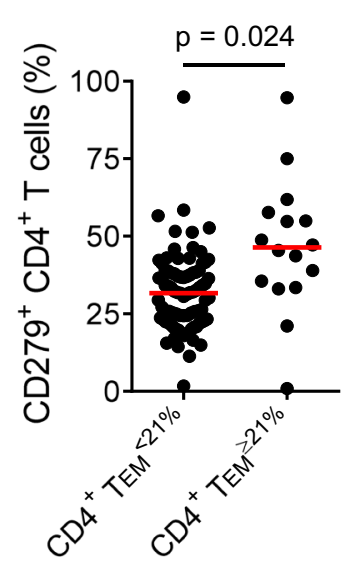

C

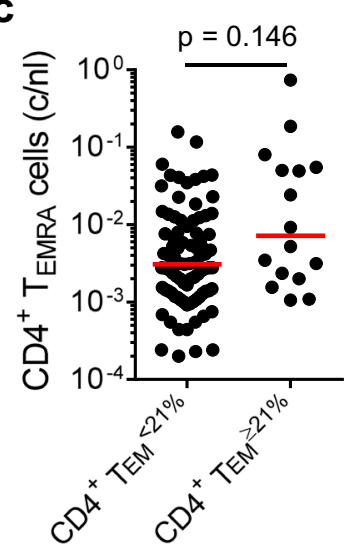

f

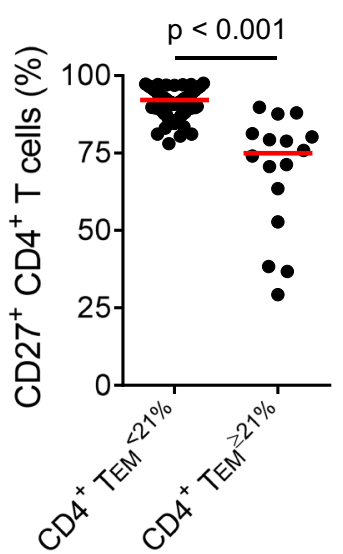

d

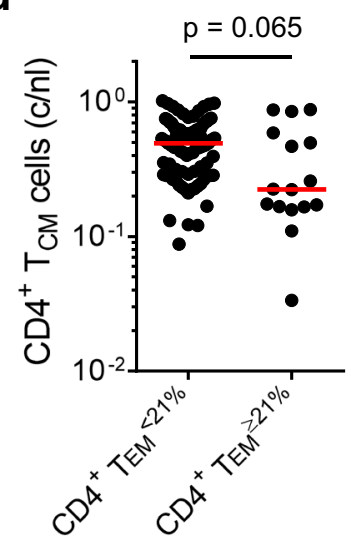

g

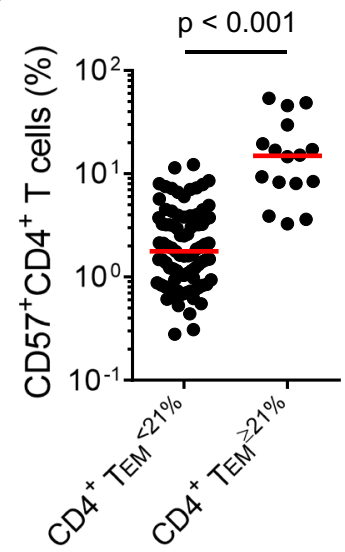

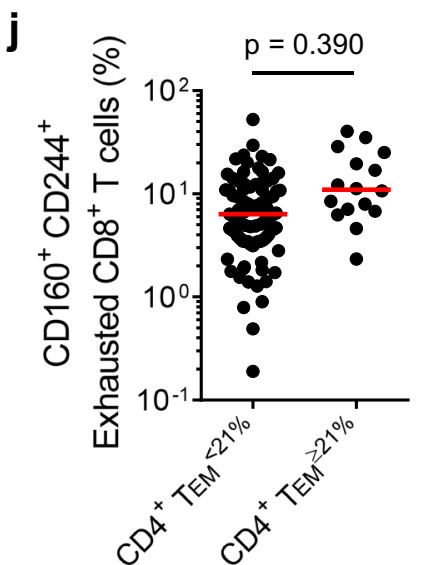

$\mathbf{k}$

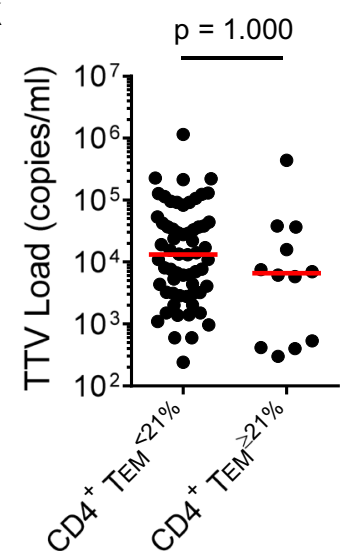

Fig. 4 CD4 ${ }^{+} \mathbf{T}_{\mathrm{EM}}$ cell enrichment is associated with chronic activation and increased numbers of effector memory CD4 $^{+} \mathbf{T}_{\text {cells. a-d }}$ Increased $\mathrm{CD} 4^{+}$ $T_{E M}$ frequency before treatment reflects a decrease in circulating naïve $C D 4^{+} T$ cells and an increase in circulating numbers of $C D 4{ }^{+} T_{E M}$ cells. a Baseline naïve CD4 ${ }^{+}$T cell counts $\left(n=103\right.$; M.W.; Bonferroni correction: $\left.m=4, p=5.2 \times 10^{-5}\right)$. b Baseline CD4 ${ }^{+} \mathrm{T}_{\mathrm{EM}}$ cell counts $(n=103$; M.W.; Bonferroni correction: $\left.m=4, p=8.0 \times 10^{-4}\right)$. c Baseline CD4 ${ }^{+} \mathrm{T}_{\text {EMRA }}$ cell counts $(n=103$; M.W.; Bonferroni-corrected $p$-value, $m=4)$. $\mathbf{d}$ Baseline CD4 ${ }^{+} \mathrm{T}_{\mathrm{CM}}$ cell counts $(n=103$; M.W.; Bonferroni-corrected $p$-value, $m=4)$. Median values are indicated by a red line. e Pairwise correlations between CD4 ${ }^{+} \mathrm{T}_{E M}$ cell frequency and other leucocyte subset frequencies in the training and validation sets $(n=103$; Pearson). Example gating strategies for analysis of flow cytometry data are provided as Supplementary Figs. 2-8. f Baseline CD27+ CD4 ${ }^{+}$T cell frequencies $(n=103 ;$ M.W.; Bonferroni correction: $m=60, p=$ $\left.4.2 \times 10^{-10}\right)$. $\mathbf{g}$ Baseline CD57+ CD4 ${ }^{+}$T cell frequencies $\left(n=103\right.$; M.W.; Bonferroni correction: $\left.m=60, p=2.0 \times 10^{-9}\right)$. h Baseline HLA-DR ${ }^{+}$CD4 ${ }^{+}$T cell frequencies $\left(n=103\right.$; M.W.; Bonferroni correction: $\left.m=60, p=4.1 \times 10^{-5}\right)$. i Baseline CD279+ CD4 + T cell frequencies $(n=103$; M.W.; Bonferronicorrected $p$-value, $m=60)$. j Baseline CD160 ${ }^{+}$CD244+ CD8 + T cell frequencies $(n=103 ;$ M.W.; Bonferroni-corrected $p$-value, $m=60)$. $\mathbf{k}$ Baseline Torque Teno Virus (TTV) loads. Uninfected patients were censored from analysis $(n=74 ;$ M.W.; Bonferroni-corrected $p$-value, $m=60)$. Median values are indicated by a red line. 
a

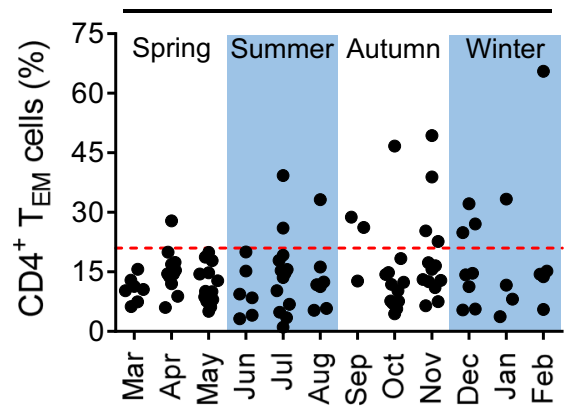

Month of $1^{\text {st }} \alpha$ PD- $1 / \alpha C T L A-4$ exposure b

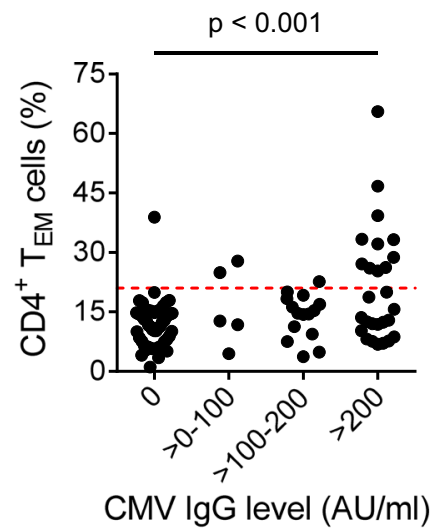

C

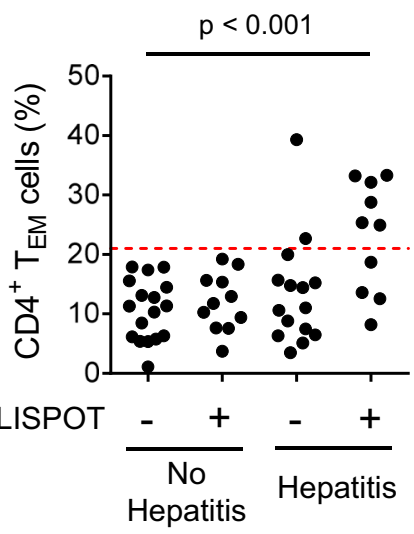

d

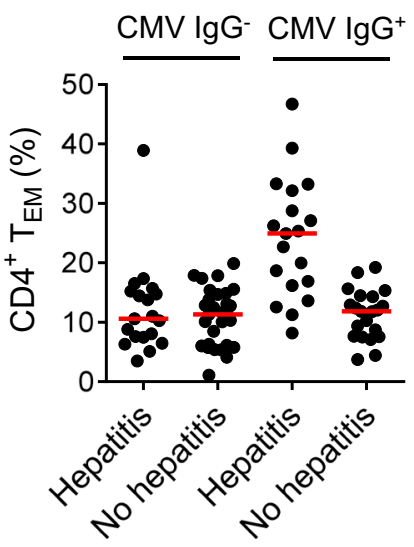

e

- All cases $(n=89)$

- $\mathrm{CMV} \mathrm{IgG}^{+}$cases $(\mathrm{n}=40)$

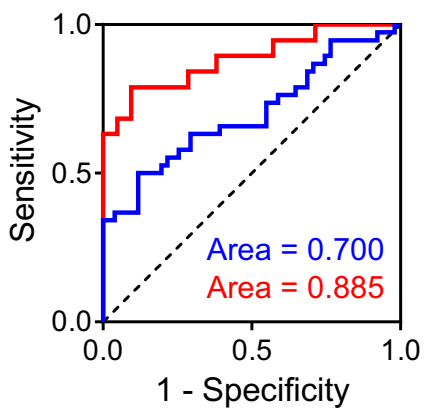

f

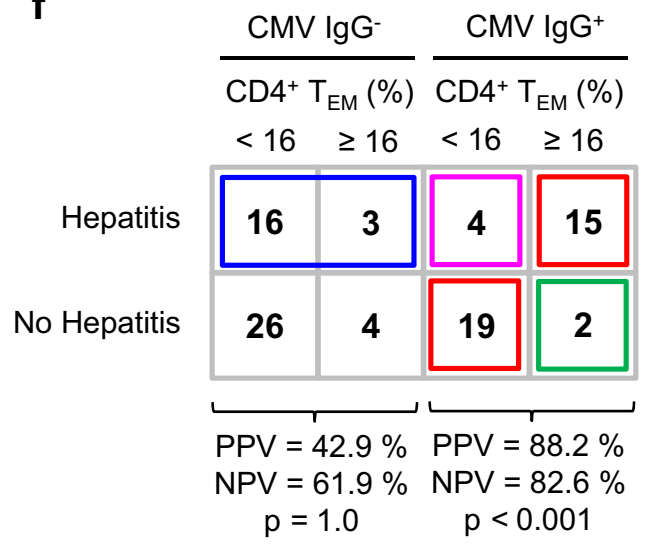

Fig. 5 CD4 ${ }^{+} \mathbf{T}_{\mathrm{EM}}$ cell expansion correlates with immunity to cytomegalovirus. a Seasonal presentation of CD4 ${ }^{+} \mathrm{T}_{\mathrm{EM}} \geq 21 \%$ patients between 2017 and 2020 ( $n=103$; F.E.). Dashed red line indicates a cut-off of CD4+ $\mathrm{T}_{\mathrm{EM}} \geq 21 \%$. $\mathbf{b}$ CD4 ${ }^{+} \mathrm{T}_{\mathrm{EM}} \geq 21 \%$ status was associated with high serum levels of anti-CMV IgG antibodies $\left(n=100 ;\right.$ F.E.; $\left.p=1.2 \times 10^{-5}\right)$. Dashed red line indicates a cut-off of CD4 ${ }^{+} \mathrm{T}_{\mathrm{EM}} \geq 21 \%$. c CD4 ${ }^{+} \mathrm{T}_{\mathrm{EM}} \geq 21 \%$ status was associated with $\mathrm{CMV}$ reactivity in pp65 ELISPOT $\left(n=53\right.$; F.E.; $\left.p=9.0 \times 10^{-5}\right)$. Dashed red line indicates a cut-off of CD4 ${ }^{+} \mathrm{T}_{\mathrm{EM}} \geq 21 \%$. d Development of hepatitis was associated with CMV-seropositivity and CD4 ${ }^{+} \mathrm{T}_{\mathrm{EM}} \geq 21 \%$ status $(n=89)$. Median values are indicated by a red line. e ROC analysis showing CD4 ${ }^{+} \mathrm{T}_{\mathrm{EM}} \%$ is a superior discriminator of patients at risk of hepatitis when considering only $\mathrm{CMV} \mathrm{IgG+}$ cases as opposed to all cases. $\mathbf{f}$ Classification of patients with unresectable metastatic melanoma who did or did not develop $\alpha$ PD-1/ $\alpha$ CTLA-4-related hepatitis according to CMV IgG status and baseline $C D 4+\mathrm{T}_{\mathrm{EM}}$ cell frequency using a revised cut-off of CD4+ $\mathrm{T}_{\mathrm{EM}} \geq 16 \%$. Red boxes indicate 34 of 40 (85\%) cases correctly classified by our model ( $n=40 ; \mathrm{F} . \mathrm{E}$; $p=1.4 \times$ $10^{-5}$ ). Green box indicates 2 of $17(11.7 \%)$ cases predicted to develop hepatitis who did not. Pink box indicates 4 of 23 (17.4\%) cases predicted not to develop hepatitis who did. Blue box indicates 19 of $49 \mathrm{CMV} \mathrm{IgG}^{-}$patients not considered by our model who developed hepatitis.

levels declined consistently from the day after valganciclovir was given, although total bilirubin continued to rise. Starting valganciclovir treatment appeared to be temporally related to increased reactivity in CMV IE-1 ELISPOT and a small decline in $\mathrm{CD}^{+}{ }^{+} \mathrm{T}_{\mathrm{EM}}$ frequency.

Liver biopsy revealed dense infiltration by lymphocytes, eosinophils and neutrophils with an accompanying ductal reaction (Fig. 8c-e). Extensive centrilobular hepatocyte necrosis was observed, which was also associated with inflammatory infiltration. These histopathological signs of significant liver damage could have been consistent with autoimmunity or viral infection. Both histological staining for CMV and CMV PCR from fixed biopsy material were negative. Although we were unable to detect virus, it is our clinical impression that markers of liver damage responded to valganciclovir treatment.

Valganciclovir prophylaxis prevents aPD-1/aCTLA-4-related hepatitis. We next asked whether valganciclovir prophylaxis could prevent $\alpha \mathrm{PD}-1 / \alpha \mathrm{CTLA}-4$-related hepatitis in predisposed individuals. The 50th, 75th and 90th percentiles of time-tohepatitis in $\mathrm{CMV}^{-\mathrm{IgG}^{+}}$patients were 24,52 and 84 days, respectively; therefore, we treated four $\mathrm{CMV} \mathrm{IgG}^{+} \mathrm{CD}_{4}^{+}$
$\mathrm{T}_{\mathrm{EM}} \geq 16 \%$ patients receiving aPD-1/aCTLA-4 therapy with prophylactic valganciclovir for between 4 and 23 weeks (Fig. 9a). Two of these patients registered mild, transient transaminitis that resolved without treatment, so were not diagnosed with hepatitis. Hence, we observed no hepatitis in the four valganciclovir-treated patients, whereas 15 of 17 (88.2\%) CMV IgG ${ }^{+} \mathrm{CD}^{+} \mathrm{T}_{\mathrm{EM}^{2}} \geq 16 \%$ patients treated with aPD-1/aCTLA-4 and no valganciclovir prophylaxis developed clinical hepatitis (Fig. 9b). To us, these four cases are consistent with the idea that CMV plays an important aetiological role in development of hepatitis after $a \mathrm{PD}$ 1/aCTLA-4 therapy in a specific subset of patients.

\section{Discussion}

Our study seeks to explain why a subset of patients with advanced melanoma is predisposed to hepatitis following immune checkpoint blockade. In our model, metastatic disease promotes compartmentalised CMV reactivation in latently infected individuals, possibly triggered by an unknown winter virus infection; in turn, CMV stimulates expansion of $\mathrm{CD}^{+} \mathrm{T}_{\mathrm{EM}}$ cells prior to immunotherapy. When aPD-1/aCTLA-4 therapy is given, these $\mathrm{T}_{\mathrm{EM}}$ cells might inflict liver injury directly or promote bystander $\mathrm{T}$ cell responses. We propose $\mathrm{CMV}$-driven $\mathrm{T}$ cell responses are a single, 
a

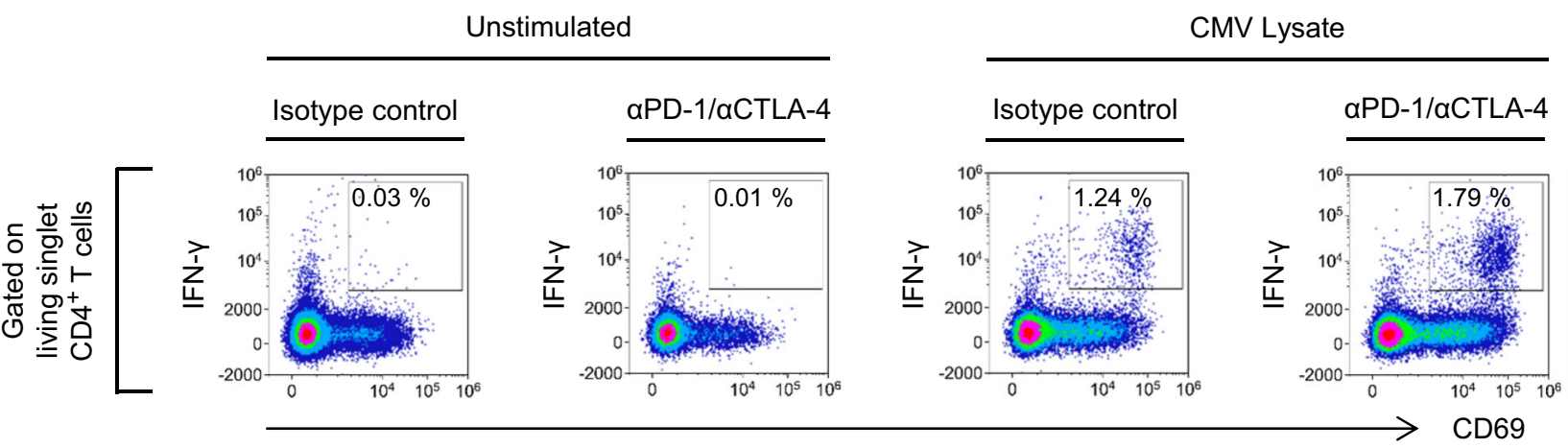

b

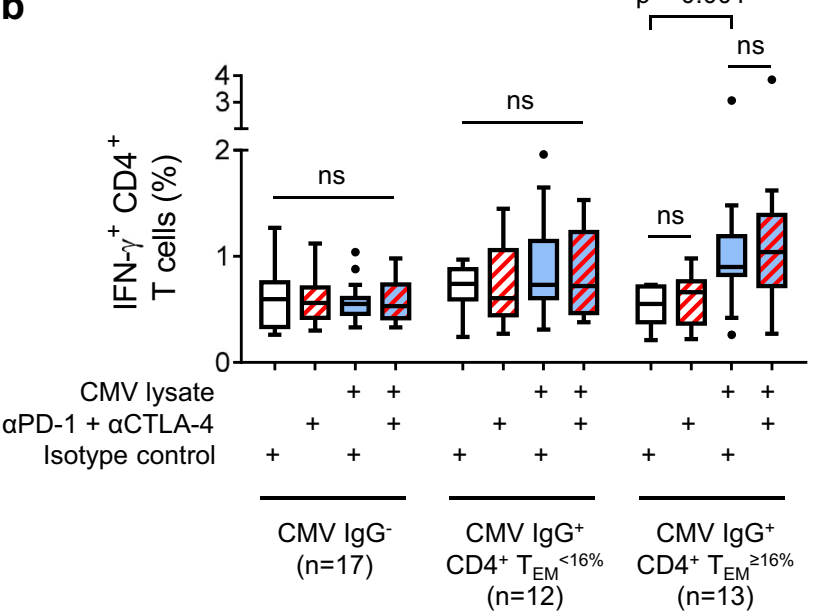

C

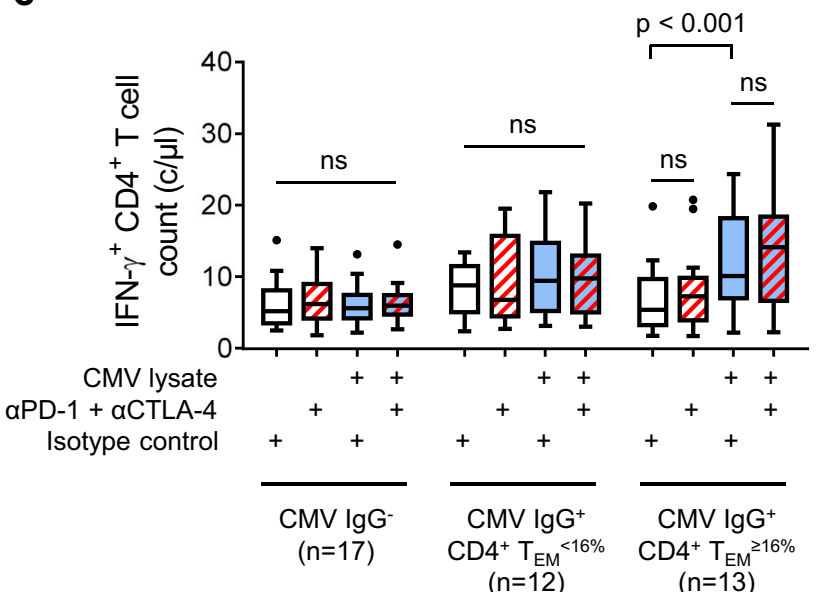

Fig. 6 CMV-reactive CD4 ${ }^{+} \mathbf{T}$ cells are enriched in patients with CD4 ${ }^{+} \mathbf{T}_{\mathrm{EM}}$ cell expansion. $\mathrm{CMV}$-reactive $\mathrm{CD} 4^{+} \mathrm{T}$ cells in patients with unresectable metastatic melanoma were assayed by in vitro stimulation with CMV lysates (blue boxes). Neutralising antibodies (red hatching) were used to detect T cells unable to respond to CMV antigens owing to expression of PD-1 or CTLA-4. T cell responses were quantified by flow cytometry analysis of cytokine expression. a Example data from a CMV IgG $+C D 4+T_{E M} \geq 16 \%$ patient who developed hepatitis. Gating of live, singlet, IFN- $\gamma$-producing $C D 4+T$ cells is illustrated in Supplementary Fig. 11. b Frequencies of IFN- $\gamma$-producing CD4+ ${ }^{+}$cells in patients categorised according to CMV IgG serostatus and baseline CD4 ${ }^{+} \mathrm{T}_{\mathrm{EM}}$ frequency $(n=42$; two-way ANOVA with Tukey correction for multiple comparisons; n.s. = not significant). Boxplots represent the median, 25th and 75th percentiles, and Tukey whiskers. c Absolute numbers of circulating IFN- $\gamma$-producing CD4 $+\mathrm{T}$ cells patients categorised according to CMV IgG serostatus and baseline CD4 ${ }^{+} \mathrm{T}_{\mathrm{EM}}$ frequency $(n=42$; two-way ANOVA with Tukey correction for multiple comparisons; n.s. $=$ not significant).

Boxplots represent the median, 25th and 75th percentiles, and Tukey whiskers.

but salient example of a general mechanism responsible for hepatitis after checkpoint blockade because other viruses or toxic agents could also drive chronic $\mathrm{CD}^{+} \mathrm{T}$ cell activation before therapy. We speculate anti-viral immunity might also explain predisposition to other adverse reactions following aPD-1/ aCTLA-4 therapy, such as colitis or pneumonitis ${ }^{37}$. The remarkable overlap between organs affected by tissue-invasive CMV disease and those susceptible to immune-related adverse reactions has not escaped our attention ${ }^{38,39}$.

Being able to identify patients predisposed to hepatitis opens various possibilities for avoiding or preventing treatment-related hepatitis ${ }^{40}$. Three of four CD4 ${ }^{+} \mathrm{T}_{\mathrm{EM}} \geq 21 \%$ patients assigned to aPD-1 monotherapy instead of aPD-1/aCTLA-4-treatment did not develop hepatitis as predicted; therefore, baseline CD4 ${ }^{+} \mathrm{T}_{\mathrm{EM}}$ expansion could be taken as a relative contraindication to dual therapy. However, a more promising strategy is to suppress occult $\mathrm{CMV}$ reactivation. In our study, valganciclovir prophylaxis apparently prevented hepatitis in four of four $\mathrm{CMV} \mathrm{IgG}^{+} \mathrm{CD}^{+}$ $\mathrm{T}_{\mathrm{EM}} \geq 16 \%$ patients. This approach must be refined in terms of dosing and duration of anti-viral therapy in randomised, controlled clinical trials, but could potentially achieve the superior outcomes of $\alpha \mathrm{PD}-1 / \alpha \mathrm{CTLA}-4$ dual therapy over $\alpha \mathrm{PD}-1$ monotherapy with fewer complications.

Because baseline $\mathrm{CD} 4{ }^{+} \mathrm{T}_{\mathrm{EM}}$ expansion was observed in patients with metastatic disease, but not in patients with fully resected melanoma, it is possible that disseminated melanoma promotes low-level CMV reactivation, perhaps through compromised $\mathrm{T}$ cell immunity, which then stimulates virus-specific $\mathrm{CD}^{+} \mathrm{T}$ cell expansion. The prominent association between $\mathrm{CD}^{+}{ }^{+} \mathrm{T}_{\mathrm{EM}}$ expansion and CMV (in contrast to other herpesgroup virus infections) might simply reflect differences in tissue tropism and, we speculate, the capacity of compartmentalised CMV reactivation in liver to drive especially strong $\mathrm{T}$ cell responses. Seasonal variation in the incidence of $\mathrm{CD} 4+\mathrm{T}_{\mathrm{EM}}$ high cases suggests that environmental factors influence expansion and contraction of the $\mathrm{CD}^{+} \mathrm{T}_{\mathrm{EM}}$ cell pool ${ }^{41,42}$. In particular, we suspect common winter viruses somehow promote CMV reactivation in liver.

Chronic, low-level exposure to viral antigens preferentially expands $\mathrm{T}_{\mathrm{EM}}$ over $\mathrm{T}_{\mathrm{CM}}{ }^{43-49}$, so $\mathrm{CMV}$-reactive $\mathrm{CD}^{+} \mathrm{T}$ cell expansion might be sustained by very little CMV replication. Ongoing $\mathrm{T}$ cell reactions against $\mathrm{CMV}$ in $\mathrm{CD} 4^{+} \mathrm{T}_{\mathrm{EM}}$ high patients are 
a

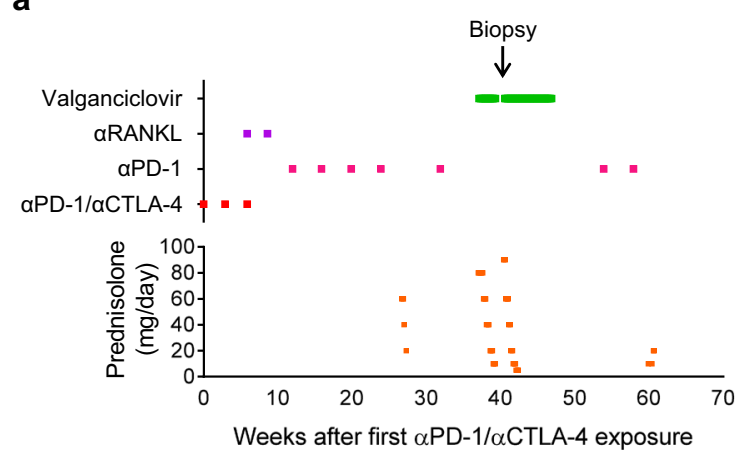

b

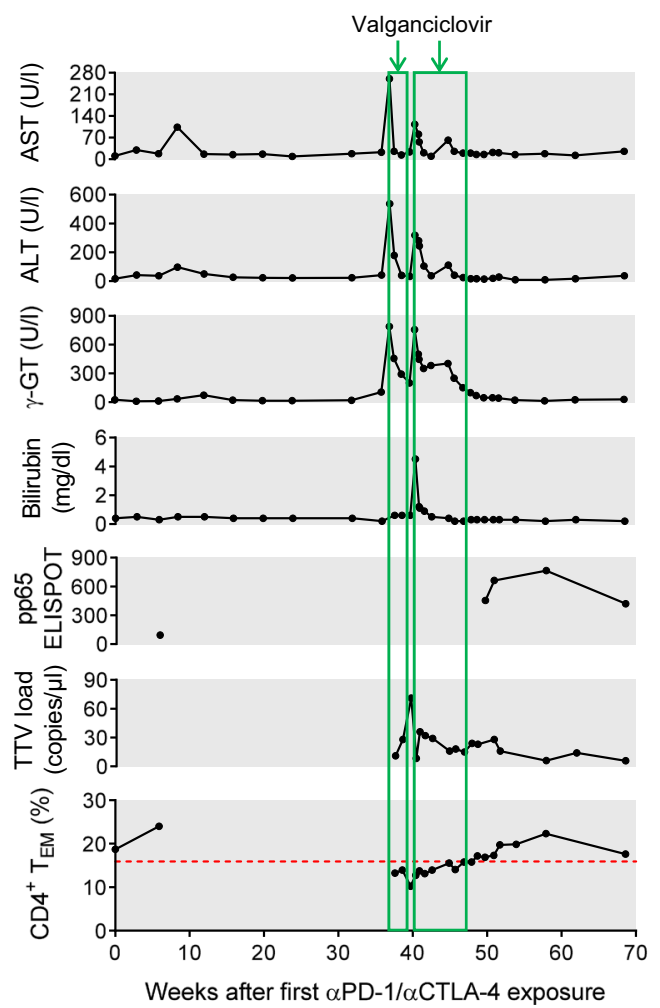

C

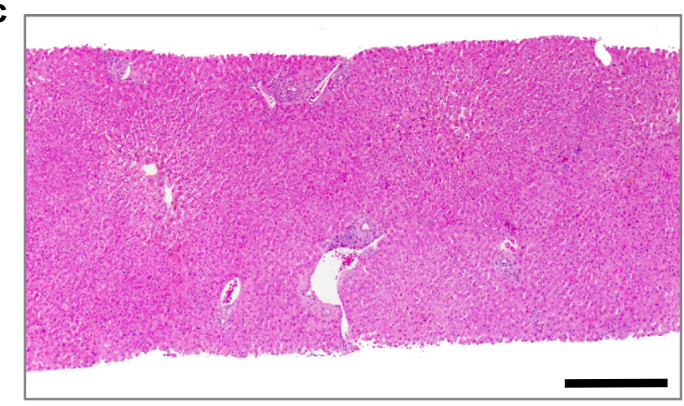

d

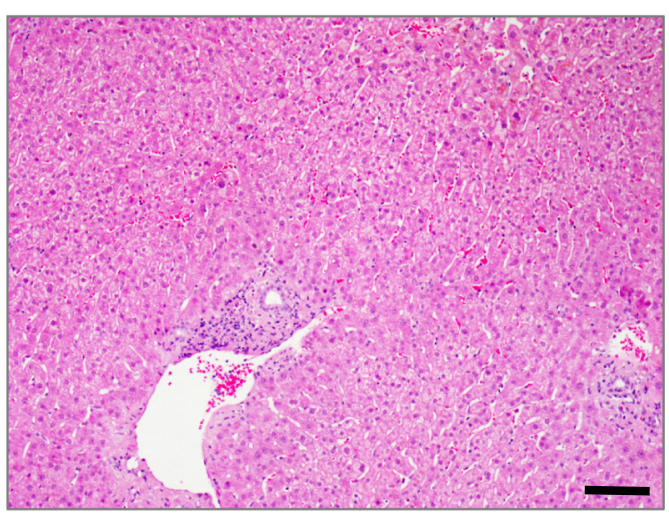

e

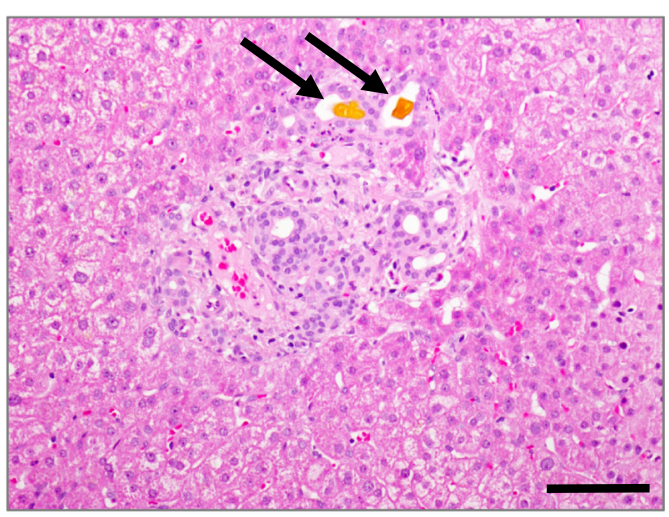

Fig. 7 Case 1: valganciclovir treatment of late-onset $\boldsymbol{\alpha P D}-\mathbf{1} / \boldsymbol{\alpha}$ CTLA-4-related hepatitis. A 54 -year-old male patient who received $\alpha$ PD-1 (Nivolumab) plus $\alpha$ CTLA-4 (Ipilimumab) dual therapy for metastatic melanoma presented with late-onset hepatitis. a Course of treatment. $\mathbf{b}$ Change in hepatitis-related parameters over time and their association with introduction, withdrawal and re-introduction of valganciclovir treatment. c The patient presented at the end of week 40 with recrudescent hepatitis and was treated with $900 \mathrm{mg} /$ day valganciclovir for 2 days prior to liver biopsy. Histopathological image of the liver biopsy (H\&E staining; scale bar $500 \mu \mathrm{m}$ ). d Generally, the liver parenchyma appeared normal (H\&E staining; scale bar $100 \mu \mathrm{m}$ ). e Only a few lymphocytes and sparse necrotic hepatocytes were observed in portal areas with small bile plugs (arrows) with no signs of hepatitis (H\&E staining; scale bar $100 \mu \mathrm{m})$.

likely to suppress CMV replication almost completely. Our inability to detect $\mathrm{CMV}$ in baseline serum samples from $\mathrm{CD} 4^{+}$ $\mathrm{T}_{\mathrm{EM}}$ high patients or liver biopsies from patients with high-grade hepatitis is a challenge for our theory; however, it may simply be beyond our technical capability to detect very low levels of compartmentalised viral reactivation. In our view, the strong correlation between CMV-specific immunity and development of hepatitis, as well as the apparent effectiveness of valganciclovir, suggests a causal role for CMV in the aetiology of $\alpha \mathrm{PD}-1 / \alpha C T L A-$ 4-related hepatitis.

We do not presently know whether CMV-specific $\mathrm{CD} 4^{+} \mathrm{T}_{\mathrm{EM}}$ cells inflict liver injury directly or provoke bystander $\mathrm{T}$ cell responses ${ }^{50}$. Administration of $\alpha$ PD-1 or aCTLA-4 has been associated with CMV reactivation in colon, so it is probable that
CMV also reactivates in liver during dual therapy ${ }^{51}$. In two patients with high-grade hepatitis, we observed liver inflammation subsided immediately after starting valganciclovir. In our view, the likely presence of viral antigen and apparent responsiveness to valganciclovir is consistent with a direct pathological effect of CMV-specific T cells, although we accept this will be hard to prove in patients.

Better understanding the contribution of anti-viral $\mathrm{T}$ cell immunity to checkpoint blockade-related adverse reactions leads us to unexpected solutions for preventing serious complications of cancer immunotherapy. From a clinical perspective, the immunological mechanisms described illustrate how patients' medical histories, especially prior exposure to pathogens, impact their experience of cancer and response to therapy. 
a

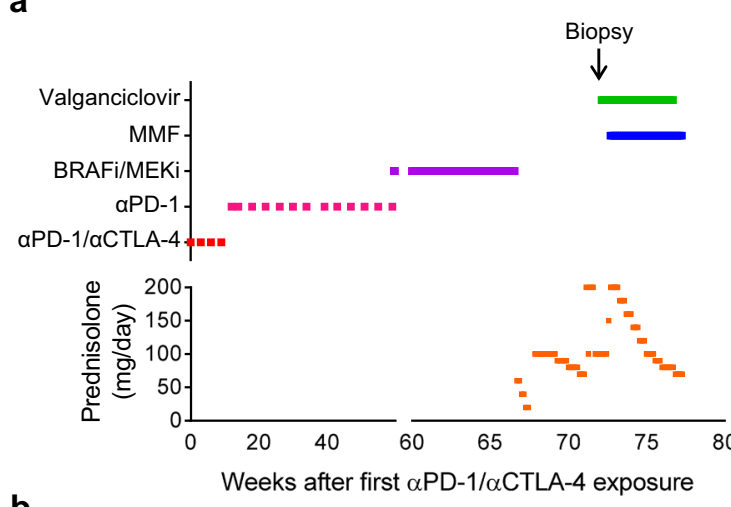

b

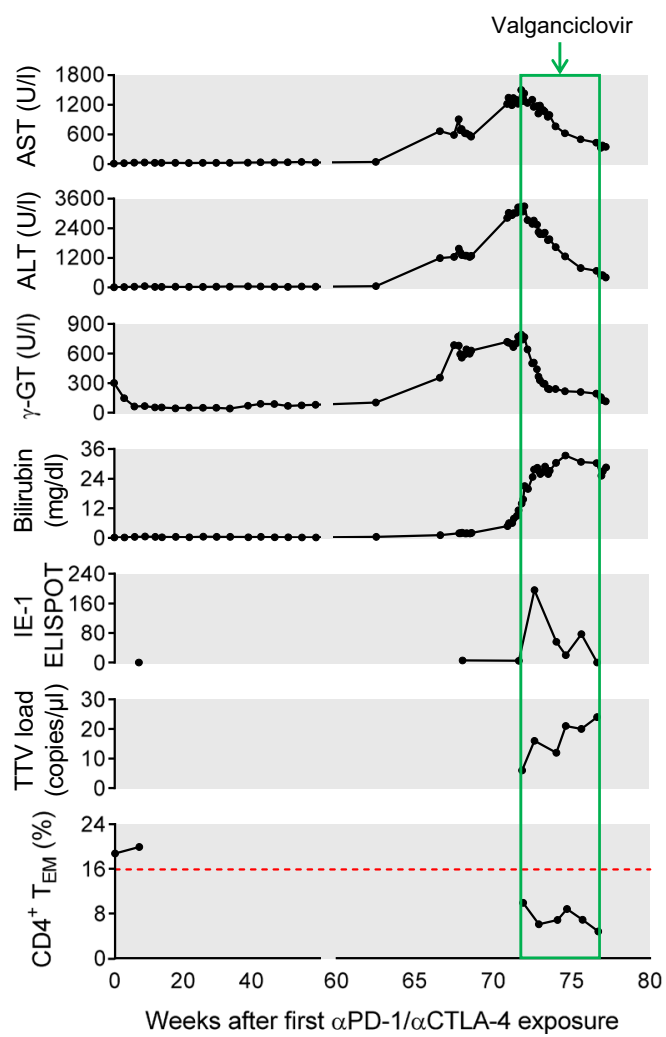

C

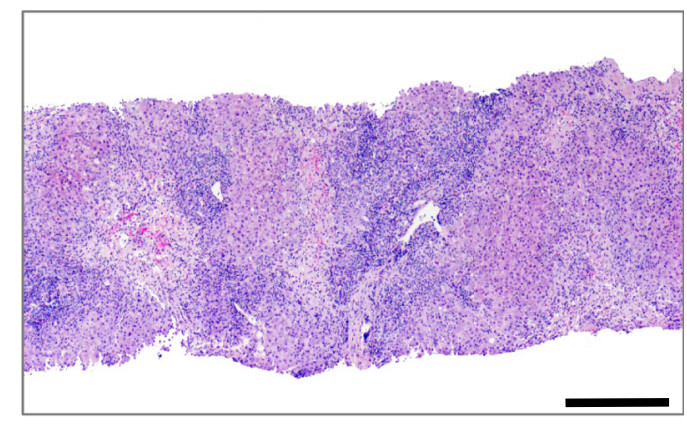

d

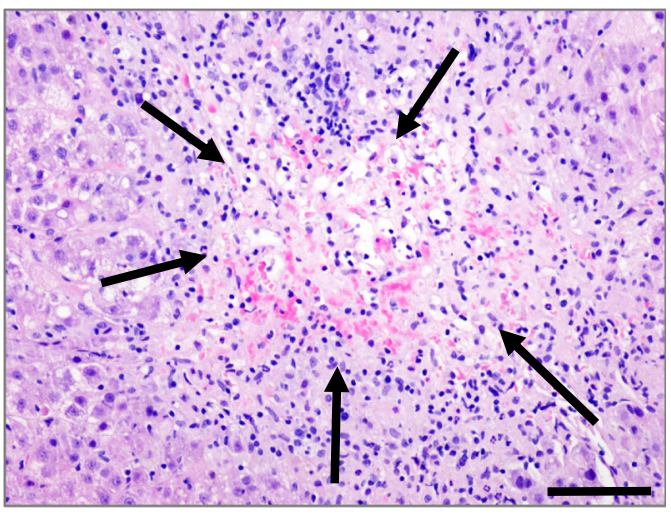

e

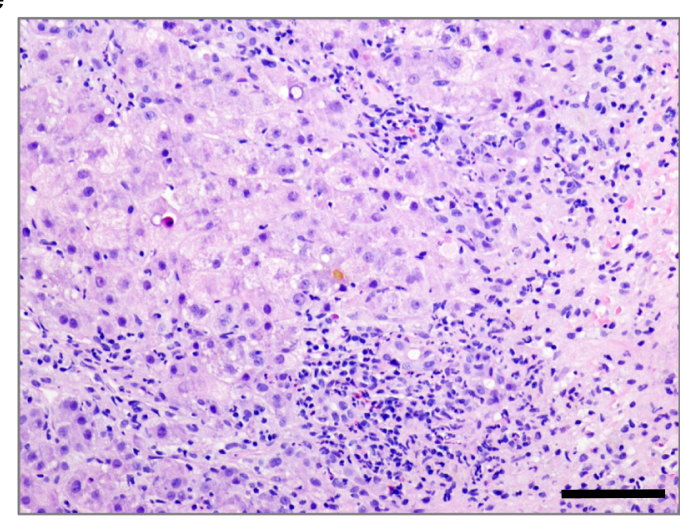

Fig. 8 Case 2: valganciclovir treatment of late-onset $\boldsymbol{\alpha P D}-\mathbf{1} / \boldsymbol{\alpha}$ CTLA-4-related hepatitis. A 49 -year-old male patient who received $\alpha$ PD-1 (Nivolumab) plus $\alpha$ CTLA-4 (Ipilimumab) dual therapy for metastatic melanoma presented with late-onset hepatitis. a Course of treatment. $\mathbf{b}$ Change in hepatitis-related parameters over time and their association with introduction of valganciclovir treatment. c Histopathological image of a liver biopsy taken at the start of week 72 showing signs consistent with drug toxicity, autoimmunity or viral infection (H\&E staining; scale bar $500 \mu \mathrm{m}$ ). d Extensive centrilobular necrosis (arrows) involving 30-40\% of hepatocytes was observed (H\&E staining; scale bar $100 \mu \mathrm{m}$ ). e Dense inflammatory infiltration of lymphocytes, eosinophils and neutrophils was seen in portal areas (H\&E staining; scale bar $100 \mu \mathrm{m}$ ).

\section{Methods}

Study approval and patient management. This study involving human research participants was performed in accordance with the Declaration of Helsinki and all applicable German and European laws and ethical standards. Specifically, specimens were obtained from patients with Stage III or IV melanoma participating in a single-centre observational clinical trial authorised by the Ethics Committee of the University of Regensburg (approval 16-101-0125) and registered with clinicaltrials. gov (NCT04158544). All participants gave full, informed written consent. The first reported patient was recruited in October 2016 and the last reported patient was recruited in July 2020. Patients received standard-of-care treatment according to local guidelines (Fig. 1a). Stage IV patients with unresectable metastatic disease who received first- or second-line checkpoint inhibitor therapy were initially treated with Nivolumab ( $\alpha$ PD-1; Bristol-Myers Squibb) and Ipilimumab ( $a$ CTLA4; Bristol-Myers Squibb) for four cycles, and thereafter with Nivolumab maintenance therapy $(3 \mathrm{mg} / \mathrm{kg}$ at 3 -week intervals). Those patients with complete resection of Stage III melanoma who received adjuvant first-line checkpoint inhibitor therapy were treated for up to 1 year with Pembrolizumab ( $\alpha$ PD-1; MSD) or Nivolumab.

Clinical flow cytometry. Detailed step-by-step protocols for preparation and analysis (including specimen gating strategies) of clinical samples by flow cytometry are available through Protocol Exchange ${ }^{52}$. In brief, peripheral blood samples were collected into ethylenediaminetetraacetic acid (EDTA)-vacutainers by venepuncture and then delivered to the immune monitoring laboratory at ambient temperature. Pre-analytical samples were stored for up to $4 \mathrm{~h}$ at $4^{\circ} \mathrm{C}$ until processing. Whole blood was stained with DuraClone reagents (Duraclone IM Phenotyping Basic Tube, B53309; Duraclone IM T cell Subsets Tube, B53328; Duraclone IM TCRs Tube, B53340; Duraclone IM Treg Tube, B53346; Duraclone IM B cells Tube, B53318; Duraclone IM Dendritic Cells Tube, B53351; all from Beckman Coulter, Krefeld, Germany). Data were recorded with a Navios ${ }^{\mathrm{TM}}$ cytometer running Cytometry List Mode Data Acquisition and Analysis Software 
aPD-1/aCTLA-4 Dual Therapy

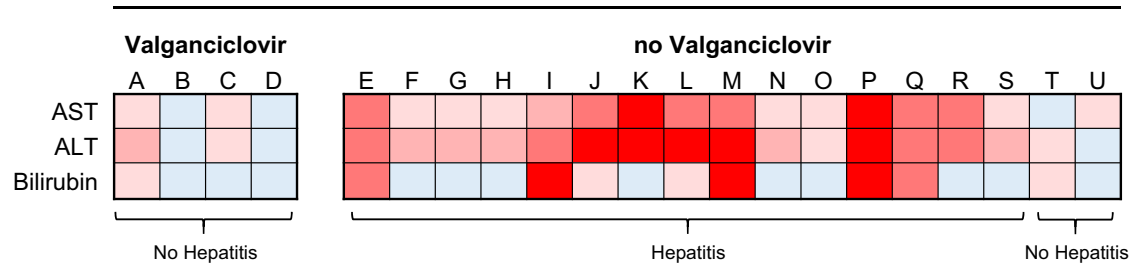

\begin{tabular}{r|c|c|c|c|c|}
\multicolumn{5}{c}{} & \multicolumn{5}{c}{ Hepatitis Severity Grade } \\
\cline { 2 - 7 } & 0 & 1 & 2 & 3 & 4 \\
\hline AST & $\leq 1$ & $>1-\leq 3$ & $>3-\leq 5$ & $>5-\leq 20$ & $>20$ \\
\cline { 2 - 6 } ALT & $\leq 1$ & $>1-\leq 3$ & $>3-\leq 5$ & $>5-\leq 20$ & $>20$ \\
\cline { 2 - 6 } Bilirubin & $\leq 1$ & $>1-\leq 1.5$ & $>1.5-\leq 3$ & $>3-\leq 10$ & $>10$ \\
\cline { 2 - 6 } & & &
\end{tabular}

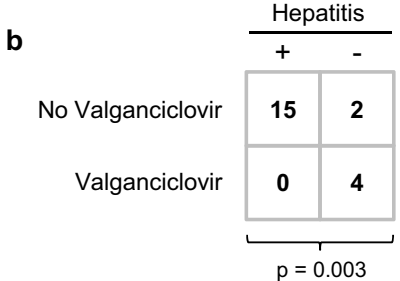

Fig. 9 Valganciclovir prophylaxis prevents $\boldsymbol{\alpha P D}-\mathbf{1 / \alpha C T L A - 4 - r e l a t e d ~ h e p a t i t i s . ~ a ~ H e a t m a p s ~ s h o w i n g ~ p e a k ~ v a l u e s ~ o f ~ A S T , ~ A L T ~ a n d ~ b i l i r u b i n ~ e x p r e s s e d ~ a s ~}$ multiples of sex-adjusted upper limit of normal ranges. Fifteen of 17 (88.2\%) patients classified as $\mathrm{CMV}_{\mathrm{IgG}}{ }^{+} \mathrm{CD}^{+} \mathrm{T}_{\mathrm{EM}} \geq 16 \%$ at baseline subsequently developed hepatitis. In contrast, 4 of $4 \mathrm{CMV} \operatorname{lgG}+\mathrm{CD}^{+} \mathrm{T}_{\mathrm{EM}} \geq 16 \%$ patients treated with $900 \mathrm{mg} /$ day prophylactic valganciclovir remained hepatitis free. b In four initial cases, prophylactic valganciclovir prevented development of hepatitis ( $n=21$; F.E.).

version 1.3 (Beckman Coulter). Blinded analyses were performed by an experienced operator using Kaluza version 2.1 according to gating strategies illustrated in Supplementary Figs. 2-8.

Detection of CMV-reactive T cells by flow cytometry. Detailed step-by-step protocols are available through Protocol Exchange ${ }^{53}$. Briefly, frozen PBMC from patients were thawed and resuspended in RPMI medium (ThermoFisher, Schwerte, Germany) supplemented with $5 \%$ heat-inactivated pooled, male-only human $A B$ serum (ZKT Tübingen, Germany), $100 \mathrm{U} / \mathrm{ml}$ penicillin, $100 \mu \mathrm{g} / \mathrm{ml}$ streptomycin and $2 \mathrm{mM}$ Glutamax (ThermoFisher). Cells were plated in 96-well round-bottom plates at $1 \times 10^{6}$ cells in $150 \mu \mathrm{l} /$ well then incubated at $37^{\circ} \mathrm{C}$ with $5 \% \mathrm{CO}_{2}$. After $6 \mathrm{~h}$, cultures were stimulated with $5 \mu \mathrm{g} / \mathrm{ml}$ CMV Lysate (Origene) and $\alpha \mathrm{CD} 28 / \alpha \mathrm{CD} 49$ for $18 \mathrm{~h}$. In some conditions, cultures were treated with neutralising monoclonal antibodies against PD-1 (MAB10864, R\&D) plus CTLA-4 (MAB3254, R\&D) at 5 $\mu \mathrm{g} / \mathrm{ml}$ each or mouse IgG1 isotype control (MAB002, R\&D) at a final concentration of $10 \mu \mathrm{g} / \mathrm{ml}$. During the last $4 \mathrm{~h}$ of cell culture, protein secretion was blocked with Protein Transport Inhibitor Cocktail (ThermoFisher) containing Brefeldin A und Monensin. Cytokine production was then assessed by flow cytometry. Dead cells were excluded with ViaKrome-808 Fixable Viability Dye (Beckman Coulter). Staining was performed in Cell Staining Buffer (BioLegend) with 10\% FcR Block (Miltenyi). Cells were fixed and permeabilised using the eBioscience IC Fixation Buffer and Permeabilization Buffer (eBiosciences, ThermoFisher). Data were recorded with a Cytoflex LX ${ }^{\mathrm{TM}}$ cytometer running CytoExpert Software (Beckman Coulter) and analysed using Kaluza version 2.1 (Beckman Coulter) according to the gating strategy shown in Supplementary Fig. 11.

18F-FDG PET/CT imaging. Hepatic glucose metabolism as a measure of liver inflammation ${ }^{34}$ was analysed retrospectively in all 32 patients who underwent pretreatment ${ }^{18} \mathrm{~F}$-FDG positron emission tomography and computed tomography (PETCT) imaging as part of routine tumour staging shortly before starting immunotherapy. After overnight fasting, whole-body PET acquisitions covering at least the trunk were started 60-90 min after i.v. injection of ${ }^{18} \mathrm{~F}$-FDG (3 MBq per kg body weight) on a Biograph Sensation 16 LSO or Biograph mCT40 Flow PET/CT scanner (both Siemens Healthcare, Germany) and lasted 18-24 min depending on patient size. The same area was covered by a low-dose CT scan (tube current $<50 \mathrm{mAs}$, tube voltage $120 \mathrm{kV}$ ) if no contrast agents were used. Otherwise $130 \mathrm{ml}$ of Accupaque ${ }^{\mathrm{TM}} 300$ (GE Healthcare) was applied as intravenous contrast agent with consecutive full-dose CT acquisition $(120 \mathrm{kV},<100 \mathrm{mAs})$. PET images (slice thickness $5 \mathrm{~mm})$ were corrected for random coincidences, decay, scatter, and attenuation and reconstructed iteratively using vendor parameter presets. PET and CT images were checked for breathing artifacts and scaled to standardised uptake values (SUV). SUV mean Blood was measured in a thread-like region-of-interest (ROI) manually set into the aorta and $\mathrm{SUV}_{\text {mean }}$ Liver in a spherical ROI in the right liver lobe using the program ROVER (Version v3.0.35, Helmholtz-Zentrum Dresden-Rossendorf, Germany). SUR was calculated as with correction for the distribution time $T$ of ${ }^{18} \mathrm{~F}$-FDG to the reference time $T_{0}$ of 75 min post injection ${ }^{54}: \mathrm{SUR}=\left(T_{0} / T\right) \times\left(\mathrm{SUV}_{\text {mean }}\right.$ liver $/ \mathrm{SUV}_{\text {mean }}$ blood $)$.

Routine clinical investigations. Routine biochemical and haematological investigations were performed by an in-house accredited diagnostic laboratory (Institute of Clinical Chemistry and Laboratory Medicine, UKR). Virological investigations were conducted by an in-house accredited laboratory (Institute of Clinical Microbiology and Hygiene, UKR) following routine diagnostic procedures. CMVreactive $\mathrm{T}$ cells were quantified by IFN- $\gamma$ ELISPOT (Lophius, Germany) according to the manufacturer's instructions in an accredited laboratory (UKR).

Statistics, data transformations and visualisation. Significance tests and curvefitting were performed with GraphPad Prism 6.04 (GraphPad Software, Inc., La Jolla, USA) or SPSS ${ }^{\circledast}$ version 25 (IBM Analytics, New York, USA). As indicated, data were analysed using a two-tailed Mann-Whitney (M.W.) test, two-tailed Fisher's exact test (F.E.), D'Agostino \& Pearson's normality test (K2), one-way or two-way ANOVA, two-tailed $t$-tests, log-rank survival analysis, receiver-operator characteristic (ROC) analysis or calculating pairwise Pearson correlations $\left(R^{2}\right)$. Where stated in the figure legends, $p$-values were adjusted for multiple comparison using Benjamini-Hochberg (B.H.) correction or Bonferroni correction, but otherwise no adjustment was made.

Reporting summary. Further information on research design is available in the Nature Research Reporting Summary linked to this article.

\section{Data availability}

Data supporting the findings of this study are available within the article and its Supplementary information files, or are available from the corresponding author subject to legal restrictions governing use of personal information. Source data are provided with this paper.

Received: 28 October 2019; Accepted: 29 January 2021; Published online: 04 March 2021

\section{References}

1. Larkin, J. et al. Five-year survival with combined nivolumab and ipilimumab in advanced melanoma. N. Engl. J. Med. 381, 1535-1546 (2019).

2. Robert, C. et al. Pembrolizumab versus ipilimumab in advanced melanoma (KEYNOTE-006): post-hoc 5-year results from an open-label, multicentre, randomised, controlled, phase 3 study. Lancet Oncol. 20, 1239-1251 (2019).

3. Robert, C. et al. Nivolumab in previously untreated melanoma without BRAF mutation. N. Engl. J. Med. 372, 320-330 (2015).

4. Postow, M. A. et al. Nivolumab and ipilimumab versus ipilimumab in untreated melanoma. N. Engl. J. Med. 372, 2006-2017 (2015).

5. Wolchok, J. D. et al. Overall survival with combined nivolumab and ipilimumab in advanced melanoma. N. Engl. J. Med. 377, 1345-1356 (2017).

6. Hodi, F. S. et al. Nivolumab plus ipilimumab or nivolumab alone versus ipilimumab alone in advanced melanoma (CheckMate 067): 4-year outcomes of a multicentre, randomised, phase 3 trial. Lancet Oncol. 19, 1480-1492 (2018).

7. Postow, M. A., Sidlow, R. \& Hellmann, M. D. Immune-related adverse events associated with immune checkpoint blockade. N. Engl. J. Med. 378, 158-168 (2018) 
8. Schadendorf, D. et al. Efficacy and safety outcomes in patients with advanced melanoma who discontinued treatment with nivolumab and ipilimumab because of adverse events: a pooled analysis of randomized phase II and III trials. J. Clin. Oncol. 35, 3807-3814 (2017).

9. Thompson, J. A. et al. Management of immunotherapy-related toxicities, version 1.2019. J. Natl Compr. Canc Netw. 17, 255-289 (2019).

10. Teufel, A. et al. Management of immune related adverse events induced by immune checkpoint inhibition. Cancer Lett. 456, 80-87 (2019).

11. Larkin, J. et al. Combined nivolumab and ipilimumab or monotherapy in untreated melanoma. N. Engl. J. Med. 373, 23-34 (2015).

12. Wolchok, J. D. et al. Nivolumab plus ipilimumab in advanced melanoma. $N$. Engl. J. Med. 369, 122-133 (2013).

13. Ruotsalainen, J. \& Tuting, T. Live or let die: $\mathrm{t}$ cell survival in cancer immunotherapy. Immunity 50, 280-282 (2019).

14. Perez-Ruiz, E. et al. Prophylactic TNF blockade uncouples efficacy and toxicity in dual CTLA-4 and PD-1 immunotherapy. Nature 569, 428-432 (2019).

15. Young, A., Quandt, Z. \& Bluestone, J. A. The balancing act between cancer immunity and autoimmunity in response to immunotherapy. Cancer Immunol. Res. 6, 1445-1452 (2018).

16. Johnson, D. B. et al. Ipilimumab therapy in patients with advanced melanoma and preexisting autoimmune disorders. JAMA Oncol. 2, 234-240 (2016).

17. Oh, D. Y. et al. Immune toxicities elicted by CTLA-4 blockade in cancer patients are associated with early diversification of the T-cell repertoire. Cancer Res. 77, 1322-1330 (2017).

18. Tivol, E. A. et al. Loss of CTLA-4 leads to massive lymphoproliferation and fatal multiorgan tissue destruction, revealing a critical negative regulatory role of CTLA-4. Immunity 3, 541-547 (1995).

19. Krishna, B. A. et al. Targeting the latent cytomegalovirus reservoir with an antiviral fusion toxin protein. Nat. Commun. 8, 14321 (2017).

20. Peghin, M. et al. Epidemiology and immediate indirect effects of respiratory viruses in lung transplant recipients: a 5-year prospective study. Am. J. Transplant. 17, 1304-1312 (2017).

21. Lancini, D., Faddy, H. M., Flower, R. \& Hogan, C. Cytomegalovirus disease in immunocompetent adults. Med. J. Aust. 201, 578-580 (2014).

22. Coussement, J. et al. When polymerase chain reaction does not help: cytomegalovirus pneumonitis associated with very low or undetectable viral load in both blood and bronchoalveolar lavage samples after lung transplantation. Transpl. Infect. Dis. 18, 284-287 (2016).

23. Almanan, M. et al. Tissue-specific control of latent CMV reactivation by regulatory T cells. PLoS Pathog. 13, e1006507 (2017)

24. Andrei, G. et al. Persistent primary cytomegalovirus infection in a kidney transplant recipient: multi-drug resistant and compartmentalized infection leading to graft loss. Antiviral Res. 168, 203-209 (2019).

25. Luck, S. E., Emery, V. C., Atkinson, C., Sharland, M. \& Griffiths, P. D. Compartmentalized dynamics of cytomegalovirus replication in treated congenital infection. J. Clin. Virol. 82, 152-158 (2016).

26. Pardieck, I. N., Beyrend, G., Redeker, A. \& Arens, R. Cytomegalovirus infection and progressive differentiation of effector-memory T cells. F1000Res 7 (2018).

27. Marchi, E., Lee, L. N. \& Klenerman, P. Inflation vs. exhaustion of antiviral CD8 + T-cell populations in persistent infections: two sides of the same coin? Front. Immunol. 10, 197 (2019).

28. Gordon, C. L. et al. Induction and maintenance of CX3CR1-intermediate peripheral memory CD8(+) T cells by persistent viruses and vaccines. Cell Rep. 23, 768-782 (2018).

29. Makwana, N. et al. CMV drives the expansion of highly functional memory $\mathrm{T}$ cells expressing NK-cell receptors in renal transplant recipients. Eur. J. Immunol. 47, 1324-1334 (2017).

30. Wei, S. C., Duffy, C. R. \& Allison, J. P. Fundamental mechanisms of immune checkpoint blockade therapy. Cancer Discov. 8, 1069-1086 (2018).

31. Tumeh, P. C. et al. PD-1 blockade induces responses by inhibiting adaptive immune resistance. Nature 515, 568-571 (2014).

32. Fujii, T. et al. Incidence of immune-related adverse events and its association with treatment outcomes: the MD Anderson Cancer Center experience. Invest. New Drugs 36, 638-646 (2018).

33. Herbst, R. S. et al. Predictive correlates of response to the anti-PD-L1 antibody MPDL3280A in cancer patients. Nature 515, 563-567 (2014).

34. Verloh, N. et al. In vivo confirmation of altered hepatic glucose metabolism in patients with liver fibrosis/cirrhosis by (18)F-FDG PET/CT. EJNMMI Res. 8, 98 (2018).

35. Khan, O. et al. TOX transcriptionally and epigenetically programs CD8(+) T cell exhaustion. Nature 571, 211-218 (2019).

36. Im, S. J. et al. Defining CD8 $+\mathrm{T}$ cells that provide the proliferative burst after PD-1 therapy. Nature 537, 417-421 (2016).

37. Caselli, E. et al. Virologic and immunologic evidence supporting an association between HHV-6 and Hashimoto's thyroiditis. PLoS Pathog. 8, e1002951 (2012).

38. Del Castillo, M. et al. The spectrum of serious infections among patients receiving immune checkpoint blockade for the treatment of melanoma. Clin. Infect. Dis. 63, 1490-1493 (2016).
39. Franklin, C. et al. Cytomegalovirus reactivation in patients with refractory checkpoint inhibitor-induced colitis. Eur. J. Cancer 86, 248-256 (2017).

40. Wilkinson, R. W. \& Leishman, A. J. Further advances in cancer immunotherapy: going beyond checkpoint blockade. Front. Immunol. 9, 1082 (2018).

41. Contreras, N. A. et al. Life-long control of cytomegalovirus (CMV) by T resident memory cells in the adipose tissue results in inflammation and hyperglycemia. PLoS Pathog. 15, e1007890 (2019).

42. Wang, Z. et al. Paradoxical effects of obesity on T cell function during tumor progression and PD-1 checkpoint blockade. Nat. Med. 25, 141-151 (2019).

43. Marzo, A. L. et al. Initial T cell frequency dictates memory CD8+ T cell lineage commitment. Nat. Immunol. 6, 793-799 (2005).

44. Wherry, E. J. et al. Lineage relationship and protective immunity of memory CD8 T cell subsets. Nat. Immunol. 4, 225-234 (2003).

45. Williams, M. A. \& Bevan, M. J. Shortening the infectious period does not alter expansion of CD8 $\mathrm{T}$ cells but diminishes their capacity to differentiate into memory cells. J. Immunol. 173, 6694-6702 (2004).

46. van Faassen, $\mathrm{H}$. et al. Reducing the stimulation of $\mathrm{CD} 8+\mathrm{T}$ cells during infection with intracellular bacteria promotes differentiation primarily into a central (CD62LhighCD44high) subset. J. Immunol. 174, 5341-5350 (2005).

47. Jelley-Gibbs, D. M. et al. Repeated stimulation of CD4 effector T cells can limit their protective function. J. Exp. Med. 201, 1101-1112 (2005).

48. Jelley-Gibbs, D. M. et al. Persistent depots of influenza antigen fail to induce a cytotoxic CD8 T cell response. J. Immunol. 178, 7563-7570 (2007).

49. Sarkar, S. et al. Strength of stimulus and clonal competition impact the rate of memory CD8 T cell differentiation. J. Immunol. 179, 6704-6714 (2007).

50. Rosato, P. C. et al. Virus-specific memory T cells populate tumors and can be repurposed for tumor immunotherapy. Nat. Commun. 10, 567 (2019).

51. Medicines and Healthcare Products Regulatory Agency. Nivolumab (Opdivo): reports of cytomegalovirus (CMV) gastrointestinal infection or reactivation. Drug Safety Update 13: No. 3. https://assets.publishing.service.gov.uk/government/ uploads/system/uploads/attachment_data/file/840565/Oct-2019-PDF.pdf (2019).

52. Kronenberg, K., Riquelme, P. \& Hutchinson, J. A. Standard protocols for immune profiling of peripheral blood leucocyte subsets by flow cytometry using DuraClone IM reagents. Protocol Exchange https://doi.org/10.21203/ rs.3.pex-757/v1 (2021)

53. Riquelme, P. \& Hutchinson, J. A. Standard protocols for detection of human CMV-specific T cells Protocol Exchange https://doi.org/10.21203/rs.3.pex-758/v1 (2021)

54. Hofheinz, F. et al. An investigation of the relation between tumor-to-liver ratio (TLR) and tumor-to-blood standard uptake ratio (SUR) in oncological FDG PET. EJNMMI Res. 6, 19 (2016).

\section{Acknowledgements}

The authors gratefully acknowledge the financial support of Bristol-Myers Squibb and the BMS Foundation (Award FA-19-009) in the form of unrestricted research grants. This study was partly supported by grants from the German Research Foundation to S.H. (DFG HA 8481/1-1) and J.A.H. (DFG HU 1838/1-1; DFG HU 1838/2-1). L.C. is a Marie Skłodowska-Curie Research Fellow affiliated with INsTRuCT and receives funding from the European Union's Horizon 2020 research and innovation programme (Award 860003). The authors thank Dr. Michael Kapinsky of Beckman Coulter GmbH for advice and support with clinical flow cytometry. The authors also thank Prof. Tobias Pukrop and staff of the Interdisciplinary Center for drug Tumor Therapy (ICT) for supporting collection of clinical samples. This work would not have been possible without the outstanding technical support of Erika Ostermeier, Marina Sorokina, Veronika Menath, Anke Hofmann and Joachim Schweimer.

\section{Author contributions}

J.A.H. designed the immune monitoring studies and other experiments, analysed the results and wrote the manuscript; K.K. organised and performed immune monitoring studies; P.R. designed and performed the experiments; J.J.W., J.K. and B.S. performed the virological analyses; K.E. provided expert histopathological descriptions; M.S., L.B. and D.H. performed the radiological and molecular imaging analyses; G.G., F.Z. and R.S. provided expert statistical and bioinformatics advice; L.C. quality-checked the manuscript; C.B. and R.B. were responsible for the routine clinical laboratory analyses; M.M. and K.D. provided the biological samples; H.L.S. and F.B. performed the experiments; H.J.S. provided infrastructural support; E.K.G., J.M.W. and S.H. provided material support, patient samples and critical feedback throughout the project. All authors approved the final version of the manuscript.

\section{Funding}

Open Access funding enabled and organized by Projekt DEAL.

\section{Competing interests}

S.H. declares he has received consulting fees and speaker's honoraria from BMS and Merck Sharp \& Dohme (MSD). All other authors declare no competing interests. 


\section{Additional information}

Supplementary information The online version contains supplementary material available at https://doi.org/10.1038/s41467-021-21572-y.

Correspondence and requests for materials should be addressed to J.A.H.

Peer review information Nature Communications thanks the anonymous reviewer(s) for their contribution to the peer review of this work. Peer reviewer reports are available.

Reprints and permission information is available at http://www.nature.com/reprints

Publisher's note Springer Nature remains neutral with regard to jurisdictional claims in published maps and institutional affiliations. (c) (i) Open Access This article is licensed under a Creative Commons Attribution 4.0 International License, which permits use, sharing, adaptation, distribution and reproduction in any medium or format, as long as you give appropriate credit to the original author(s) and the source, provide a link to the Creative Commons license, and indicate if changes were made. The images or other third party material in this article are included in the article's Creative Commons license, unless indicated otherwise in a credit line to the material. If material is not included in the article's Creative Commons license and your intended use is not permitted by statutory regulation or exceeds the permitted use, you will need to obtain permission directly from the copyright holder. To view a copy of this license, visit http://creativecommons.org/licenses/by/4.0/.

(c) The Author(s) 2021, corrected publication 2021 